\title{
Degree growth of birational maps of the plane
}

\author{
JÉRÉMY BLANC AND JULIE DÉSERTI
}

\begin{abstract}
This article studies the sequence of iterative degrees of a birational map of the plane. This sequence is known either to be bounded or to have a linear, quadratic or exponential growth.

The classification elements of infinite order with a bounded sequence of degrees is achieved, the case of elements of finite order being already known. The coefficients of the linear and quadratic growth are then described, and related to geometrical properties of the map. The dynamical number of base-points is also studied.

Applications of our results are the description of embeddings of the BaumslagSolitar groups and $\mathrm{GL}(2, \mathbb{Q})$ into the Cremona group.
\end{abstract}

Mathematics Subject Classification (2010): 14E07 (primary); 37F10, 32H50 (secondary).

\section{Introduction}

A rational map of the complex projective plane $\mathbb{P}^{2}=\mathbb{P}_{\mathbb{C}}^{2}$ into itself is a map of the following type

$$
\phi: \mathbb{P}^{2} \rightarrow \mathbb{P}^{2}, \quad(x: y: z) \rightarrow\left(\phi_{0}(x, y, z): \phi_{1}(x, y, z): \phi_{2}(x, y, z)\right),
$$

where the $\phi_{i}$ 's are homogeneous polynomials of the same degree without common factor. The degree $\operatorname{deg} \phi$ of $\phi$ is by definition the degree of these polynomials. We will only consider birational maps, which are rational maps having a rational inverse, and denote by $\operatorname{Bir}\left(\mathbb{P}^{2}\right)$ the group of such maps, classically called Cremona group.

We are interested in the behaviour of the sequence $\left\{\operatorname{deg} \phi^{k}\right\}_{k \in \mathbb{N}}$. According to [13], the sequence is either bounded or has a linear, quadratic or exponential growth. We will say that $\phi$ is

(1) elliptic if the growth is bounded;

(2) a Jonquières twist if the growth is linear;

Both authors were supported by the Swiss National Science Foundation grant no PP00P2_128422/1. Received June 7, 2012; accepted in revised version April 23, 2013. 
(3) a Halphen twist if the growth is quadratic;

(4) hyperbolic if the growth is exponential.

This terminology is classical, and consistent with the natural action of $\operatorname{Bir}\left(\mathbb{P}^{2}\right)$ on a hyperbolic space of infinite dimension, where Jonquières and Halphen twists are parabolic ([9, Theorem 3.6]). Recall that a Jonquières twist preserves a unique pencil of rational curves [13, Theorem 0.2], and that this one can be sent by a birational map of the plane onto a pencil of lines ([15, Section 43]). This explains the terminology, since an element of $\operatorname{Bir}\left(\mathbb{P}^{2}\right)$ which preserves a pencil of rational curves is classically called Jonquières transformation. A Halphen twist preserves a unique pencil of elliptic curves ([14], [13, Theorem 0.2, Lemma 4.1]) and this one can be sent by a birational map of the plane onto a pencil of curves of degree $3 n$ with 9 points of multiplicity $n$ ([15, Section 43]). These pencils have been studied by Halphen ([16]) and are now called Halphen pencils; this again explains the choice of the terminology.

Recall that the first dynamical degree of $\phi \in \operatorname{Bir}\left(\mathbb{P}^{2}\right)$ is $\lambda(\phi)=\lim _{k \rightarrow+\infty}\left(\operatorname{deg} \phi^{k}\right)^{1 / k} \in$ $\mathbb{R}$. This is an invariant of conjugation which allows to distinguish the first three cases (where $\lambda(\phi)=1$ ) from the last case (where $\lambda(\phi)>1$ ).

The nature of the growth is invariant under conjugation, and induces geometric properties on $\phi$, that we describe now.

It is shown in [13, Theorem 0.2, Lemma 4.1] that an element $\phi \in \operatorname{Bir}\left(\mathbb{P}^{2}\right)$ is elliptic if and only if it is conjugate to an automorphism $g \in \operatorname{Aut}(\mathrm{S})$ of a smooth projective rational surface $S$ such that $g^{n}$ belongs to the connected component $\operatorname{Aut}^{0}(\mathrm{~S})$ of Aut(S) for some $n>0$. We refine this result by showing that we can choose $g$ of finite order or $\mathrm{S}=\mathbb{P}^{2}$ (and hence $n=1$ ). The complete classification of elements of finite order of $\operatorname{Bir}\left(\mathbb{P}^{2}\right)$ can be found in [8]; for elliptic elements of infinite order, one has (Proposition 2.3):

Theorem A. If $\phi \in \operatorname{Bir}\left(\mathbb{P}^{2}\right)$ is elliptic of infinite order, then $\phi$ is conjugate to an automorphism of $\mathbb{P}^{2}$, which restricts to one of the following automorphisms on some open subset isomorphic to $\mathbb{C}^{2}$ :

(1) $(x, y) \mapsto(\alpha x, \beta y)$, where $\alpha, \beta \in \mathbb{C}^{*}$, and where the kernel of the group homomorphism $\mathbb{Z}^{2} \rightarrow \mathbb{C}^{*}$ given by $(i, j) \mapsto \alpha^{i} \beta^{j}$ is generated by $(k, 0)$ for some $k \in \mathbb{Z}$;

(2) $(x, y) \mapsto(\alpha x, y+1)$, where $\alpha \in \mathbb{C}^{*}$.

The end of Section 2 is devoted to the description of the conjugacy classes of such maps (Proposition 2.4) and their centralisers in the Cremona group (Lemmas 2.7 and 2.8).

A birational map $\phi \in \operatorname{Bir}\left(\mathbb{P}^{2}\right)$ has a finite number $\mathfrak{b}(\phi)$ of base-points (that may belong to $\mathbb{P}^{2}$ or correspond to infinitely near points). We will call the number

$$
\mu(\phi)=\lim _{k \rightarrow+\infty} \frac{\mathfrak{b}\left(\phi^{k}\right)}{k},
$$


the dynamical number of base-points of $\phi$. In Section 3, we study the sequence $\left\{\mathfrak{b}\left(\phi^{k}\right)\right\}_{k \in \mathbb{N}}$ and deduce some properties on the number $\mu(\phi)$. In particular, we show that $\mu(\phi)$ is a non-negative integer invariant under conjugation; it also allows us to give a characterisation of birational maps conjugate to an automorphism of a smooth projective rational surface (Proposition 3.5):

Theorem B. Let $\mathrm{S}$ be a smooth projective surface; the birational map $\phi \in \operatorname{Bir}(\mathrm{S})$ is conjugate to an automorphism of a smooth projective surface if and only if $\mu(\phi)=0$.

In the case where $\phi$ is a Jonquières twist, the number $\mu(\phi)$ determines the degree growth of $\phi$. The sequence $\left\{\operatorname{deg} \phi^{k}\right\}_{k \in \mathbb{N}}$ grows as $\alpha k$ for some constant $\alpha \in \mathbb{R}$. The number $\alpha$ is not invariant under conjugation, but one can show that the minimal value is attained when the rational curves of the unique invariant pencil of curves are lines. More precisely, one has (Proposition 4.5):

Theorem C. Let $\phi \in \operatorname{Bir}\left(\mathbb{P}^{2}\right)$ be a Jonquières twist.

(1) The set

$$
\left\{\lim _{k \rightarrow+\infty} \frac{\operatorname{deg}\left(\psi \phi^{k} \psi^{-1}\right)}{k} \mid \psi \in \operatorname{Bir}\left(\mathbb{P}^{2}\right)\right\}
$$

admits a minimum, which is equal to $\frac{\mu(\phi)}{2} \in \frac{1}{2} \mathbb{N}$.

(2) There exists an integer $a \in \mathbb{N}$ such that

$$
\lim _{k \rightarrow+\infty} \frac{\operatorname{deg}\left(\phi^{k}\right)}{k}=\frac{\mu(\phi)}{2} \cdot a^{2} .
$$

Moreover, $a=1$ if and only if $\phi$ preserves a pencil of lines.

In the case of Halphen twists we also find a similar result (Proposition 5.1):

Theorem D. Let $\phi \in \operatorname{Bir}\left(\mathbb{P}^{2}\right)$ be a Halphen twist.

(1) The set

$$
\left\{\lim _{k \rightarrow+\infty} \frac{\operatorname{deg}\left(\psi \phi^{k} \psi^{-1}\right)}{k^{2}} \mid \psi \in \operatorname{Bir}\left(\mathbb{P}^{2}\right)\right\}
$$

admits a minimum $\kappa(\phi) \in \mathbb{Q}_{>0}$.

(2) There exists an integer $a \geq 3$ such that

$$
\lim _{k \rightarrow+\infty} \frac{\operatorname{deg}\left(\phi^{k}\right)}{k^{2}}=\kappa(\phi) \cdot \frac{a^{2}}{9} .
$$

Moreover, $a=3$ if and only if $\phi$ preserves a Halphen pencil. 
An application of our results is the description of birational maps whose two distinct iterates are conjugate, the non-existence of embeddings of Baumslag-Solitar groups into the Cremona group and the description of the embeddings of $\mathrm{GL}(2, \mathbb{Q})$ into the Cremona group (Section 6):

Theorem E. Let $\phi$ denote a birational map of $\mathbb{P}^{2}$ of infinite order. Assume that $\phi^{n}$ and $\phi^{m}$ are conjugate and that $|m| \neq|n|$. Then, $\phi$ is conjugate to an automorphism of $\mathbb{C}^{2}$ of the form $(x, y) \mapsto(\alpha x, y+1)$, where $\alpha \in \mathbb{C}^{*}$ such that $\alpha^{m+n}=1$ or $\alpha^{m-n}=1$.

In particular, if $\phi$ is conjugate to $\phi^{n}$ for any positive integer $n$, then $\phi$ is conjugate to $(x, y) \mapsto(x, y+1)$.

Theorem $\mathbf{F}$. If $|m|,|n|, 1$ are distinct, there is no embedding of

$$
\mathrm{BS}(m, n)=\left\langle r, s \mid r s^{m} r^{-1}=s^{n}\right\rangle, \quad m, n \in \mathbb{Z}, m n \neq 0
$$

into the Cremona group.

Theorem G. Let $\rho: \operatorname{GL}(2, \mathbb{Q}) \rightarrow \operatorname{Bir}\left(\mathbb{P}^{2}\right)$ be an embedding. Up to conjugation by an element of $\operatorname{Bir}\left(\mathbb{P}^{2}\right)$, there exists an odd integer $k$ and a homomorphism $\chi: \mathbb{Q}^{*} \rightarrow$ $\mathbb{C}^{*}$ (with respect to multiplication) such that

$$
\rho\left(\left[\begin{array}{ll}
a & b \\
c & d
\end{array}\right]\right)=\left(x \cdot \frac{\chi(a d-b c)}{(c y+d)^{k}}, \frac{a y+b}{c y+d}\right), \quad \forall\left[\begin{array}{ll}
a & b \\
c & d
\end{array}\right] \in \mathrm{GL}(2, \mathbb{Q}) .
$$

Remark 1.1. All these statements hold replacing $\mathbb{C}$ by an algebraically closed field of characteristic zero.

ACKNOWLEDGEMENTS. The authors would like to thank the referee for his helpful remarks and suggestions. Thanks also to Yves de Cornulier and Ivan Marin for interesting discussions on group theory, to Charles Favre for questions on elliptic maps of infinite order and to Serge Cantat for his remarks and references.

\section{Elliptic maps}

\subsection{Classification of elliptic maps of infinite order}

If $\phi$ is a birational map of $\mathbb{P}^{2}$ such that $\left\{\operatorname{deg} \phi^{k}\right\}_{k \in \mathbb{N}}$ is bounded, it is conjugate to an automorphism $g$ of a smooth rational surface $S$ such that the action of $g$ on $\operatorname{Pic}(\mathrm{S})$ is finite [13, Lemma 4.1]. If $g$ has finite order, the possible conjugacy classes are completely classified in [8]. Here we deal with the case of elements of infinite order, classifying the possibilities and describing its centralisers in $\operatorname{Bir}\left(\mathbb{P}^{2}\right)$. 
Proposition 2.1. Let $g$ be an automorphism of a smooth rational surface $\mathrm{S}$ which has infinite order but has a finite action on $\mathrm{Pic}(\mathrm{S})$. Then, there exists a birational morphism $\mathrm{S} \rightarrow \mathrm{X}$ where $\mathrm{X}$ is equal either to $\mathbb{P}^{2}$ or to a Hirzebruch surface $\mathbb{F}_{n}$ for $n \neq 1$, which conjugates $\mathrm{g}$ to an automorphism of $\mathrm{X}$.

Remark 2.2. The proof of this result follows from a study of possible minimal pairs, which is similar to the one made in [7] for finite abelian subgroups of $\operatorname{Bir}\left(\mathbb{P}^{2}\right)$ (see [7, Lemmas 3.2, 6.1, 9.7]).

Proof. Contracting the possible sets of disjoint $(-1)$-curves on $\mathrm{S}$ which are invariant by $g$, we can assume that the action of $g$ on $\mathrm{S}$ is minimal. The action of $g$ on Pic(S) being of finite order, the process corresponds to applying a $G$-Mori program, where $G$ is a finite group acting on $\operatorname{Pic}(\mathrm{S})$ (we only look at parts of the Picard group which are invariant). Denoting by $\mathrm{Pic}(\mathrm{S})^{g} \subset \mathrm{Pic}(\mathrm{S})$ the submodule $\{L \in \operatorname{Pic}(\mathrm{S}) \mid g(L)=L\}$, one of the following occurs $([17,18])$ :

(1) $\operatorname{Pic}(\mathrm{S})^{g}$ has rank 1 and $\mathrm{S}$ is a del Pezzo surface;

(2) $\operatorname{Pic}(\mathrm{S})^{g}$ has rank 2 , and there exist a conic bundle $\pi: S \rightarrow \mathbb{P}^{1}$ on $\mathrm{S}$, together with an automorphism $h$ of $\mathbb{P}^{1}$ such that $h \circ \pi=\pi \circ g$.

We want to show that $\mathrm{S}$ is $\mathbb{P}^{2}$ or a Hirzebruch surface $\mathbb{F}_{n}$ for $n \neq 1$, and exclude the other cases.

In the case where $\mathrm{Pic}(\mathrm{S})^{g}$ has rank 1, the fact that $g$ has infinite order but finite action on Pic(S) implies that the kernel of the group homomorphism Aut(S) $\rightarrow$ $\operatorname{Aut}(\operatorname{Pic}(\mathrm{S}))$ is infinite. So $\mathrm{S}$ is a del Pezzo surface of degree $\left(K_{\mathrm{S}}\right)^{2} \geq 6$. The surface cannot be $\mathbb{F}_{1}$ otherwise the exceptional section would be invariant. Similarly, it cannot be the unique del Pezzo surface of degree 7, which has exactly three $(-1)$ curves, forming a chain (one touches the two others, which are disjoint), because the curve of the middle (and also the union of the two others) would be invariant. The only possibilities are thus $\mathbb{P}^{2}, \mathbb{P}^{1} \times \mathbb{P}^{1}=\mathbb{F}_{0}$, and the del Pezzo surface of degree 6 .

If $\mathrm{S}$ is the del Pezzo surface of degree 6 , any element $h \in \operatorname{Aut}(\mathrm{S})$ acting minimally on S has finite order [7, Lemma 9.7]. Let us recall the simple argument. The del Pezzo surface of degree 6 is isomorphic to

$$
\mathrm{S}=\left\{(x: y: z),(u: v: w) \in \mathbb{P}^{2} \times \mathbb{P}^{2} \mid x u=y v=z w\right\} .
$$

The projections $\pi_{1}, \pi_{2}$ on each factor are birational morphisms contracting three $(-1)$-curves on $p_{1}=(1: 0: 0), p_{2}=(0: 1: 0)$ and $p_{3}=(0: 0: 1)$. The group Pic(S) is generated by the six $(-1)$-curves of S, which are $E_{i}=\left(\pi_{1}\right)^{-1}\left(p_{i}\right)$ and $F_{i}=\left(\pi_{2}\right)^{-1}\left(p_{i}\right)$ for $1 \leq i \leq 3$ and form an hexagon. In fact, the action on the hexagon gives rise to an isomorphism $\operatorname{Aut}(\mathrm{S}) \simeq\left(\mathbb{C}^{*}\right)^{2} \rtimes\left(\mathrm{Sym}_{3} \rtimes \mathbb{Z} / 2 \mathbb{Z}\right)=$ $\left(\mathbb{C}^{*}\right)^{2} \rtimes D_{6}$. The action of $g$ on $\mathrm{S}$ being minimal, $g$ permutes cyclically the curves, and either $g$ or $g^{-1}$ acts as $\left(E_{1} \rightarrow F_{2} \rightarrow E_{3} \rightarrow F_{1} \rightarrow E_{2} \rightarrow F_{3}\right)$. This implies that $g$ or $g^{-1}$ is equal to

$$
((x: y: z),(u: v: w)) \rightarrow((\alpha v: \beta w: u),(\beta y: \alpha z: \alpha \beta x)),
$$

for some $\alpha, \beta \in \mathbb{C}^{*}$, and has order 6 . 
We can now assume the existence of an invariant conic bundle $\pi: S \rightarrow \mathbb{P}^{1}$. If $\pi$ has no singular fibre, then $\mathrm{S}$ is a Hirzebruch surface $\mathbb{F}_{n}$ and $n \neq 1$ because of the minimality of the action. It remains to exclude the case where $\pi$ has at least one singular fibre. The minimality of the action on $\mathrm{S}$ implies that the two components of every singular fibre $F$ (which are two $(-1)$-curves) are exchanged by a power $g^{k}$ of $g$, and in particular that the whole singular fibre is invariant by $g^{k}$. Note that $k$ a priori depends on $F$.

We now prove that $g^{k}$ does not act trivially on the base of the conic bundle, i.e. that $g^{k}$ sends a general fibre onto another one. If $g^{k}$ acts trivially on the base of the fibration, the automorphism $g^{2 k}$ acts trivially on Pic(S); taking a birational morphism $\mathrm{S} \rightarrow \mathbb{F}_{n}$ which contracts a component in each singular fibre one conjugates $g^{2 k}$ to an automorphism of $\mathbb{F}_{n}$, which fixes pointwise at least one section. The strict transform on $\mathrm{S}$ of this section intersects only one component in each singular fibre and its image by $g^{k}$ gives thus another section, also fixed by $g^{2 k}$. The action of $g^{k}$ on a general fibre of $\pi$ exchanges the two points of the two sections and hence has order 2: contradiction.

The action of $g^{k}$ on the base is non-trivial and fixes the point of $\mathbb{P}^{1}$ corresponding to the singular fibre; so the same holds for $g$ (recall that the fixed points of an element of $\operatorname{Aut}\left(\mathbb{P}^{1}\right)$ and any of its non-trivial powers are the same). This implies that $F$ is invariant by $g$, so its two components are exchanged by it (and thus $k$ is odd).

In particular, $g$ exchanges the two components of every singular fibre. This implies that the number of singular fibres of $\pi$ is at most 2 , so $S$ is the blow-up of one or two points of a Hirzebruch surface.

The fact that the two components of at least one singular fibre are exchanged gives a symmetry on the sections, that will help us to determine S. Denote by $-m$ the minimal self-intersection of a section of $\pi$ and let $s$ be one section which realises this minimum. Contracting the components in the singular fibres which do not intersect $s$, one has a birational morphism $S \rightarrow \mathbb{F}_{n}$. The image of $s$ is a section with minimal self-intersection, so $m=n$. If $n=0$, then taking some section of $\mathbb{F}_{0}=\mathbb{P}^{1} \times \mathbb{P}^{1}$ of self-intersection 0 passing through at least one blown-up point, its strict transform on $\mathrm{S}$ would be a section of negative self-intersection, which contradicts the minimality of $s^{2}$, so $m=n>0$. Let us denote by $s^{\prime}$ the section $g(s)$, which also has self-intersection $-m$ on $\mathrm{S}$ but self-intersection $-m+r$ on $\mathbb{F}_{m}$, where $r$ is the number of singular fibres of $\pi$. Because any section of $\mathbb{F}_{m}$ distinct from the exceptional section has self-intersection $\geq m$, we get $-m+r \geq m$, so $2 \geq r \geq 2 m$, which implies that $m=1$ and $r=2$.

The surface $S$ is thus the blow-up of two points on $\mathbb{F}_{1}$, not lying on the exceptional section and not on the same fibre, so is a del Pezzo surface of degree 6 . The fact that $g$ acts minimally on $\mathrm{S}$ is impossible, as we already observed.

Proposition 2.3. Let $\phi$ be a birational map of $\mathbb{P}^{2}$ of infinite order, such that $\left\{\operatorname{deg} \phi^{k}\right\}_{k \in \mathbb{N}}$ is bounded.

Then $\phi$ is conjugate to an automorphism of $\mathbb{P}^{2}$, which restricts to one of the following automorphisms on some open subset isomorphic to $\mathbb{C}^{2}$ : 
(1) $(x, y) \mapsto(\alpha x, \beta y)$, where $\alpha, \beta \in \mathbb{C}^{*}$, and where the kernel of the group homomorphism $\mathbb{Z}^{2} \rightarrow \mathbb{C}^{*}$ given by $(i, j) \mapsto \alpha^{i} \beta^{j}$ is generated by $(k, 0)$ for some $k \in \mathbb{Z}$;

(2) $(x, y) \mapsto(\alpha x, y+1)$, where $\alpha \in \mathbb{C}^{*}$.

Proof. According to Proposition 2.1 the map $\phi$ is conjugate to an automorphism of a minimal surface $S$, equal to either $\mathbb{P}^{2}$ or a Hirzebruch surface.

Suppose first that $S=\mathbb{P}^{2}$. Looking at the Jordan normal form, any automorphism of $\mathbb{P}^{2}$ is conjugate to

- either $(x: y: z) \mapsto(\alpha x: \beta y: z)$,

- or $(x: y: z) \mapsto(\alpha x: y+z: z)$,

- or $(x: y: z) \mapsto(x+y: y+z: z)$.

This latter automorphism is conjugate to $(x: y: z) \mapsto(x: y+z: z)$ in $\operatorname{Bir}\left(\mathbb{P}^{2}\right)$ (for instance by $(x: y: z) \rightarrow\left(x z-\frac{1}{2} y(y-z): y z: z^{2}\right)$, as already observed in [6, Example 1]). It remains to study the case of diagonal automorphisms to show the assertion on the kernel stated in the proposition. As in the proof of [6, Proposition 6], we associate to each diagonal automorphism $\psi:(x: y: z) \mapsto$ $(\alpha x: \beta y: z)$ the kernel $\Delta_{\psi}$ of the following homomorphism of groups:

$$
\delta_{\psi}: \mathbb{Z}^{2} \rightarrow \mathbb{C}^{*}, \quad(i, j) \mapsto \alpha^{i} \beta^{j} .
$$

For any $M=\left[\begin{array}{ll}a & b \\ c & d\end{array}\right] \in \mathrm{GL}(2, \mathbb{Z})$, we denote by $M(\psi)$ the diagonal automorphism

$$
(x: y: z) \mapsto\left(\alpha^{a} \beta^{b} x: \alpha^{c} \beta^{d} y: z\right),
$$

which is the conjugate of $\psi$ by the birational map $(x, y) \rightarrow\left(x^{a} y^{b}, x^{c} y^{d}\right)$ (viewed in the chart $z=1$ ). We can check that

$$
\delta_{M(\psi)}=\delta_{\psi} \circ{ }^{t} M
$$

which implies that $\Delta_{M(\psi)}={ }^{t} M^{-1}\left(\Delta_{\psi}\right)$. We can always choose $M$ (by a result on Smith's normal form) such that $\Delta_{M(\phi)}$ is generated by $k_{1} e_{1}$ and $k_{1} k_{2} e_{2}$, where $e_{1}$, $e_{2}$ are the canonical basis vectors of $\mathbb{Z}^{2}$, and $k_{1}, k_{2}$ are non-negative integers, and replace $\phi$ with $M(\phi)$, which is conjugate to it. Since $\phi$ and $M(\phi)$ have infinite order, we see that $k_{2}=0$, and get the assertion on the kernel stated in the proposition.

If $S=\mathbb{F}_{0}=\mathbb{P}^{1} \times \mathbb{P}^{1}$, we can reduce to the case of $\mathbb{P}^{2}$ by blowing-up a fixed point and contracting the strict transform of the members of the two rulings passing through the point.

Suppose now that $S=\mathbb{F}_{n}$ for $n \geq 2$. If $g$ fixes a point of $\mathbb{F}_{n}$ which is not on the exceptional section, we can blow-up the point and contract the strict transform of the fibre to go to $\mathbb{F}_{n-1}$. We can thus assume that all points of $\mathbb{F}_{n}$ fixed by $g$ are on the exceptional section. The action of $g$ on the base of the fibration is, up to conjugation, $x \mapsto \alpha x$ or $x \mapsto x+1$ for some $\alpha \in \mathbb{C}^{*}$. Removing the fibre at 
infinity and the exceptional section, we get $\mathbb{C}^{2}$, where the action of $g$ is

- either $(x, y) \mapsto(\alpha x, \beta y+Q(x))$,

- or $(x, y) \mapsto(x+1, \beta y+Q(x))$,

where $\alpha, \beta \in \mathbb{C}^{*}$ and $Q$ is a polynomial of degree $\leq n$.

The action on the fibre at infinity is obtained by conjugating by $(x, y) \rightarrow-\rightarrow$ $\left(\frac{1}{x}, \frac{y}{x^{n}}\right)$

In the first case, there is no fixed point on the fibre at infinity (except the point on the exceptional section) if and only if $\beta=\alpha^{n}$ and $\operatorname{deg} Q=n$. There is no fixed point on $x=0$ if and only if $Q(0) \neq 0$ and $\beta=1$. This implies that $\alpha$ is a primitive $k$-th rooth of unity, where $k$ is a divisor of $n$. Conjugating by $\left(x, y+\gamma x^{d}\right)$ we replace $Q(x)$ with $Q(x)+\gamma\left(\alpha^{d}-1\right) x^{d}$, so we can assume that the coefficient of $x^{d}$ is trivial if $d$ is not a multiple of $k$, which means that $Q(x)=P\left(x^{k}\right)$ for some polynomial $P \in \mathbb{C}[x]$. In particular, $g$ is equal to $(x, y) \mapsto\left(\xi x, y+P\left(x^{k}\right)\right)$ and is conjugate to $(x, y) \mapsto(\xi x, y+1)$ by $(x, y) \rightarrow-\rightarrow\left(x, \frac{y}{P\left(x^{k}\right)}\right)$.

In the second case, there is no fixed point on $\mathbb{C}^{2}$, and no point on the fibre at infinity if and only if $\beta=1$ and $\operatorname{deg} Q=n$. Conjugating $g$ by $(x, y) \mapsto$ $\left(x, y+\gamma x^{n+1}\right)$ (which corresponds to performing an elementary link $\mathbb{F}_{n}-\rightarrow \mathbb{F}_{n+1}$ at the unique fixed-point and then coming back with an elementary link at a general point of the fibre at infinity), we get

$$
(x, y) \mapsto\left(x+1, y-\gamma x^{n+1}+Q(x)+\gamma(x+1)^{n+1}\right) .
$$

Choosing the right element $\gamma \in \mathbb{C}$, we can decrease the degree of $Q(x)$, and get $(x, y) \mapsto(x+1, y)$ by induction.

\subsection{Conjugacy classes of elliptic maps of infinite order}

Following [6], we will call elements of the form $(x, y) \mapsto(\alpha x, \beta y)$, respectively $(x, y) \mapsto(\alpha x, y+1)$ diagonal automorphisms, respectively almost-diagonal automorphisms of $\mathbb{C}^{2}$ (or $\mathbb{P}^{2}$ ). The conjugacy classes in each family are given by the following:

\section{Proposition 2.4 ([6], Theorem 1).}

(1) A diagonal automorphism and an almost-diagonal automorphism of $\mathbb{C}^{2}$ are never conjugate in $\operatorname{Bir}\left(\mathbb{C}^{2}\right)$.

(2) Two diagonal automorphisms $(x, y) \mapsto(\alpha x, \beta y)$ and $(x, y) \mapsto(\gamma x, \delta y)$ are conjugate in $\operatorname{Bir}\left(\mathbb{C}^{2}\right)$ if and only if there exists $\left[\begin{array}{ll}a & b \\ c & d\end{array}\right] \in \mathrm{GL}(2, \mathbb{Z})$ such that $\left(\alpha^{a} \beta^{b}, \alpha^{c} \beta^{d}\right)=(\gamma, \delta)$.

(3) Two almost diagonal automorphisms $(x, y) \mapsto(\alpha x, y+1)$ and $(x, y) \mapsto$ $(\gamma x, y+1)$ are conjugate in $\operatorname{Bir}\left(\mathbb{C}^{2}\right)$ if and only if $\alpha=\gamma^{ \pm 1}$. 
Corollary 2.5. Let $\phi \in \operatorname{Bir}\left(\mathbb{P}^{2}\right)$ be an elliptic map which has infinite order. If $\phi^{m}$ is conjugate to $\phi^{n}$ in $\operatorname{Bir}\left(\mathbb{P}^{2}\right)$ for some $m, n \in \mathbb{Z},|m| \neq|n|$, then $\phi$ is conjugate to an automorphism of $\mathbb{C}^{2}$ of the form $(x, y) \mapsto(\alpha x, y+1)$, where $\alpha \in \mathbb{C}^{*}$ such that $\alpha^{m+n}=1$ or $\alpha^{m-n}=1$.

Proof. Note that $m n \neq 0$ since $\phi$ has infinite order. Then $\phi$ is conjugate to one of the two cases of Proposition 2.3.

First of all, assume that up to conjugation $\phi$ is $(x, y) \mapsto(\alpha x, \beta y)$ and that the kernel $\Delta_{\phi}$ of the group homomorphism $\mathbb{Z}^{2} \rightarrow \mathbb{C}^{*}$ given by $(i, j) \mapsto \alpha^{i} \beta^{j}$ is generated by $(k, 0)$ for some $k \in \mathbb{Z}$. Since $\phi^{m}$ and $\phi^{n}$ are conjugate there exists a matrix

$$
N=\left[\begin{array}{ll}
a & b \\
c & d
\end{array}\right] \in \mathrm{GL}(2, \mathbb{Z})
$$

such that $\left(\left(\alpha^{m}\right)^{a}\left(\beta^{m}\right)^{b},\left(\alpha^{m}\right)^{c}\left(\beta^{m}\right)^{d}\right)=\left(\alpha^{n}, \beta^{n}\right)$ (Proposition 2.4). This means that

$$
\left(\alpha^{m a-n} \beta^{m b}, \alpha^{m c} \beta^{m d-n}\right)=(1,1),
$$

so $(m a-n, m b),(m c, m d-n)$ belong to $\Delta_{\phi}$. In particular $m b=m d-n=0$, which implies that $b=0$, so $a d= \pm 1$, which is impossible since $m \neq \pm n$.

Assume now that $\phi$ is conjugate to $(x, y) \mapsto(\alpha x, y+1)$ for some $\alpha$ in $\mathbb{C}^{*}$. The fact that $\phi^{m}$ and $\phi^{n}$ are conjugate implies that $\alpha^{m+n}=1$ or $\alpha^{m-n}=1$ (Proposition 2.4).

\subsection{Centralisers of elliptic maps of infinite order}

If $\phi$ is a birational map of $\mathbb{P}^{2}$, we will denote by $\mathrm{C}(\phi)$ the centraliser of $\phi$ in $\operatorname{Bir}\left(\mathbb{P}^{2}\right)$ :

$$
\mathrm{C}(\phi)=\left\{\psi \in \operatorname{Bir}\left(\mathbb{P}^{2}\right) \mid \phi \psi=\psi \phi\right\} .
$$

In the sequel, we describe the centralisers of elliptic maps of infinite order of $\operatorname{Bir}\left(\mathbb{P}^{2}\right)$. The results are groups which contain the centralisers of some elements of PGL $(2, \mathbb{C})$. We recall the following result, whose proof is an easy exercise. Recall that PGL $(2, \mathbb{C})$ is the group of automorphisms of $\mathbb{P}^{1}$, or equivalently the group of Möbius transformations $x \rightarrow-\frac{a x+b}{c x+d}$.

Lemma 2.6. For any $\alpha \in \mathbb{C}^{*}$, we have

$$
\{\eta \in \operatorname{PGL}(2, \mathbb{C}) \mid \eta(\alpha x)=\alpha \eta(x)\}= \begin{cases}\operatorname{PGL}(2, \mathbb{C}) & \text { if } \alpha=1 \\ \left\{x--\gamma x^{ \pm 1} \mid \gamma \in \mathbb{C}^{*}\right\} & \text { if } \alpha=-1 \\ \left\{x \mapsto \gamma x \mid \gamma \in \mathbb{C}^{*}\right\} & \text { if } \alpha \neq \pm 1 .\end{cases}
$$

Lemma 2.7. Let us consider $\phi:(x, y) \mapsto(\alpha x, \beta y)$ where $\alpha, \beta$ are in $\mathbb{C}^{*}$, and where the kernel of the group homomorphism $\mathbb{Z}^{2} \rightarrow \mathbb{C}^{*}$ given by $(i, j) \mapsto \alpha^{i} \beta^{j}$ is generated by $(k, 0)$ for some $k \in \mathbb{Z}$. Then the centraliser of $\phi$ in $\operatorname{Bir}\left(\mathbb{P}^{2}\right)$ is $\mathrm{C}(\phi)=\left\{(x, y) \rightarrow\left(\eta(x), y R\left(x^{k}\right)\right) \mid R \in \mathbb{C}(x), \eta \in \operatorname{PGL}(2, \mathbb{C}), \eta(\alpha x)=\alpha \eta(x)\right\}$. 
Proof. Let $\psi:(x, y) \rightarrow\left(\psi_{1}(x, y), \psi_{2}(x, y)\right)$ be an element of $\mathrm{C}(\phi)$. The fact that $\psi$ commutes with $\phi$ is equivalent to

$(\star) \psi_{1}(\alpha x, \beta y)=\alpha \psi_{1}(x, y) \quad$ and $\quad(\diamond) \psi_{2}(\alpha x, \beta y)=\beta \psi_{2}(x, y)$.

Writing $\psi_{i}=\frac{P_{i}}{Q_{i}}$ for $i=1,2$, where $P_{i}, Q_{i}$ are polynomials without common factors, we see that $P_{1}, P_{2}, Q_{1}, Q_{2}$ are eigenvectors of the linear automorphism $\phi^{*}$ of the $\mathbb{C}$-vector space $\mathbb{C}[x, y]$ given by $\phi^{*}: f(x, y) \mapsto f(\alpha x, \beta y)$. This means that each of the $P_{i}, Q_{i}$ is a product of a monomial in $x, y$ with an element of $\mathbb{C}\left[x^{k}\right]$. Using $(\star)$ and $(\diamond)$, we get the existence of $R_{1}, R_{2} \in \mathbb{C}(x)$ such that

$$
\psi_{1}(x, y)=x R_{1}\left(x^{k}\right), \quad \psi_{2}(x, y)=y R_{2}\left(x^{k}\right) .
$$

The fact that $\psi$ is birational implies that $\psi_{1}(x, y)$ is an element $\eta(x) \in \operatorname{PGL}(2, \mathbb{C})$; it satisfies $\eta(\alpha x)=\alpha \eta(x)$ because of $(\star)$.

Lemma 2.8. Let us consider $\phi:(x, y) \mapsto(\alpha x, y+\beta)$ where $\alpha, \beta \in \mathbb{C}^{*}$. The centraliser of $\phi$ in $\operatorname{Bir}\left(\mathbb{P}^{2}\right)$ is equal to

$$
\begin{array}{r}
\mathrm{C}(\phi)=\{(x, y) \rightarrow(\eta(x), y+R(x)) \mid \eta \in \operatorname{PGL}(2, \mathbb{C}), \\
\eta(\alpha x)=\alpha \eta(x), R \in \mathbb{C}(x), R(\alpha x)=R(x)\} .
\end{array}
$$

Proof. Conjugating by $(x, y) \mapsto(x, \beta y)$, we can assume that $\beta=1$.

Let $\psi:(x, y) \rightarrow\left(\psi_{1}(x, y), \psi_{2}(x, y)\right)$ be a birational map of $\mathbb{P}^{2}$ which commutes with $\phi$. One has

$$
(\star) \psi_{1}(\alpha x, y+1)=\alpha \psi_{1}(x, y) \quad \text { and } \quad(\diamond) \psi_{2}(\alpha x, y+1)=\psi_{2}(x, y)+1 .
$$

Equality $(\star)$ implies that $\psi_{1}$ only depends on $x$ (see [6, Lemma 2]). Therefore $\psi_{1}$ is an element of PGL(2, $\mathbb{C})$ which commutes with $x \mapsto \alpha x$.

It follows from $(\diamond)$ that

$$
\frac{\partial \psi_{2}}{\partial y}(\alpha x, y+1)=\frac{\partial \psi_{2}}{\partial y}(x, y) \quad \text { and } \quad \frac{\partial \psi_{2}}{\partial x}(\alpha x, y+1)=\alpha^{-1} \frac{\partial \psi_{2}}{\partial x}(x, y) \text {, }
$$

which again means that $\frac{\partial \psi_{2}}{\partial x}(x, y)$ and $\frac{\partial \psi_{2}}{\partial y}(x, y)$ only depend on $x$. The second component of $\psi$ can thus be written $a y+B(x)$, where $a \in \mathbb{C}^{*}, B \in \mathbb{C}(x)$. Replacing this form in $(\diamond)$, we get

$$
B(\alpha x)=B(x)+1-a,
$$

which implies that $\frac{\partial B}{\partial x}(\alpha x)=\alpha^{-1} \frac{\partial B}{\partial x}(x)$, and thus that $x \frac{\partial B}{\partial x}(x)$ is invariant under $x \mapsto \alpha x$.

If $\alpha$ is not a root of unity, this means that $\frac{\partial B}{\partial x}=c / x$ for some $c \in \mathbb{C}$; since $B$ is a rational function, one gets $c=0$ and $B$ is a constant (or equivalently an element such that $B(\alpha x)=B(x))$. It implies moreover $a=1$ and we are done.

If $\alpha$ is a primitive $k$-th root of unity, the fact that $\psi:(x, y) \rightarrow(\eta(x), a y+$ $B(x))$ commutes with

$$
\phi^{k}:(x, y) \rightarrow(x, y+k)
$$

yields $a(y+k)+B(x)=a y+B(x)+k$, so $a=1$. We again get $B(\alpha x)=B(x)$. 


\section{On the growth of the number of base-points}

If $\mathrm{S}$ is a projective smooth surface, every $\phi \in \operatorname{Bir}(\mathrm{S})$ admits a resolution

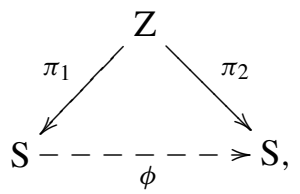

where $\pi_{1}, \pi_{2}$ are sequences of blow-ups. The resolution is minimal if and only if no $(-1)$-curve of $Z$ is contracted by both $\pi_{1}$ and $\pi_{2}$. The base-points of $\phi$ are the points blown-up by $\pi_{1}$, which can be points of $S$ or infinitely near points. We denote by $\mathfrak{b}(\phi)$ the number of such points, which is also equal to the difference of the ranks of $\operatorname{Pic}(Z)$ and $\operatorname{Pic}(\mathrm{S})$, and thus equal to $\mathfrak{b}\left(\phi^{-1}\right)$.

We will call the number

$$
\mu(\phi)=\lim _{k \rightarrow+\infty} \frac{\mathfrak{b}\left(\phi^{k}\right)}{k},
$$

the dynamical number of base-points of $\phi$. Since $\mathfrak{b}(\phi \psi) \leq \mathfrak{b}(\phi)+\mathfrak{b}(\psi)$ for any $\phi$, $\psi \in \operatorname{Bir}(\mathrm{S})$, we see that $\mu(\phi)$ is a non-negative real number. Moreover, $\mathfrak{b}\left(\phi^{-1}\right)$ and $\mathfrak{b}(\phi)$ being always equal, we get $\mu\left(\phi^{k}\right)=|k \cdot \mu(\phi)|$ for any $k \in \mathbb{Z}$. We can also make the following easy observation:

Lemma 3.1. The dynamical number of base-points is an invariant of conjugation: if $\theta: \mathrm{S} \rightarrow \mathrm{Z}$ is a birational map between smooth projective surfaces and $\phi \in$ $\operatorname{Bir}(\mathrm{S})$, then

$$
\mu(\phi)=\mu\left(\theta \phi \theta^{-1}\right) .
$$

In particular, if $\phi$ is conjugate to an automorphism of a smooth projective surface, then $\mu(\phi)=0$.

Proof. We have

$$
-2 \mathfrak{b}(\theta)+\mathfrak{b}\left(\phi^{n}\right) \leq \mathfrak{b}\left(\theta \phi^{n} \theta^{-1}\right) \leq 2 \mathfrak{b}(\theta)+\mathfrak{b}\left(\phi^{n}\right) \text { for all } n \in \mathbb{Z}
$$

This yields $\mu(\phi)=\mu\left(\theta \phi \theta^{-1}\right)$. It is thus clear that $\mu(\phi)=0$ if $\phi$ is conjugate to an automorphism of a smooth projective surface.

In this section, we precise the properties of the number $\mu$, and will in particular see that it is an integer.

If $\phi \in \operatorname{Bir}(\mathrm{S})$ is a birational map, we will say that a (possibly infinitely near) base-point $p$ of $\phi$ is a persistent base-point if there exists an integer $N$ such that $p$ is a base-point of $\phi^{k}$ for any $k \geq N$ but is not a base-point of $\phi^{-k}$ for any $k \geq N$. 
If $p$ is a point of $\mathrm{S}$ or a point infinitely near, which is not a base-point of $\phi \in \operatorname{Bir}(\mathrm{S})$, we define a point $\phi^{\bullet}(p)$, which will also be a point of $\mathrm{S}$ or a point infinitely near. For this, take a minimal resolution

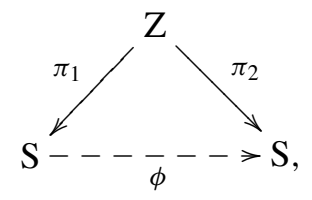

where $\pi_{1}, \pi_{2}$ are sequences of blow-ups. Because $p$ is not a base-point of $\phi$, it corresponds, via $\pi_{1}$, to a point of $Z$ or infinitely near. Using $\pi_{2}$, we view this point on S, again maybe infinitely near, and call it $\phi^{\bullet}(p)$.

Remark 3.2. If $p$ is not a base-point of $\phi \in \operatorname{Bir}(\mathrm{S})$ and $\phi(p)$ is not a base-point of $\psi \in \operatorname{Bir}(\mathbf{S})$, we have $(\psi \phi)^{\bullet}(p)=\psi^{\bullet}\left(\phi^{\bullet}(p)\right)$. If $p$ is a general point of S, then $\phi^{\bullet}(p)=\phi(p) \in S$.

Example 3.3. If $\mathrm{S}=\mathbb{P}^{2}, p=(1: 0: 0)$ and $\phi$ is the birational map $(x: y: z)-\rightarrow$ $\left(y z+x^{2}: x z: z^{2}\right)$, the point $\phi^{\bullet}(p)$ is not equal to $p=\phi(p)$, but is infinitely near to it.

Using this definition, we put an equivalence relation on the set of points that belong to $\mathrm{S}$ or are infinitely near, by saying that $p$ is equivalent to $q$ if there exists an integer $k$ such that $\left(\phi^{k}\right)^{\bullet}(p)=q$ (this implies that $p$ is not a base-point of $\phi^{k}$ and that $q$ is not a base-point of $\phi^{-k}$ ). The set of equivalence classes is the generalisation of the notion of set of orbits for birational maps.

Proposition 3.4. Let $\phi$ be a birational map of a smooth projective surface S. Denote by $v$ the number of equivalence classes of persistent base-points of $\phi$. Then, the set

$$
\left\{\mathfrak{b}\left(\phi^{k}\right)-\nu k \mid k \geq 0\right\} \subset \mathbb{Z}
$$

is bounded.

In particular, $\mu(\phi)$ is an integer, equal to $\nu$.

Proof. Let us say that a base-point $q$ is periodic if $\left(\phi^{k}\right)^{\bullet}(q)=q$ for some $k \neq 0$, or if $q$ is a base-point of $\phi^{k}$ for any $k \in \mathbb{Z} \backslash\{0\}$ (which implies that $\left(\phi^{k}\right)^{\bullet}(q)$ is never defined for $k \neq 0$ ). Let us denote by $\mathcal{P}$ the set of periodic base-points of $\phi$ and by $\widehat{\mathcal{P}}$ the finite set of points equivalent to a point of $\mathcal{P}$.

The number of base-points of $\phi$ and $\phi^{-1}$ being finite, there exists an integer $N$ such that for any non-periodic base-point $p$ and for any $j, j^{\prime} \geq N, p$ is a base-point of $\phi^{j}$ (respectively of $\phi^{-j}$ ) if and only if $p$ is a base-point of $\phi^{j^{\prime}}$ (respectively of $\phi^{-j^{\prime}}$ ). 
We decompose the set of non-periodic base-points of $\phi$ into four sets:

$\mathcal{B}_{++}=\left\{p \mid p\right.$ is a base-point of $\phi^{j}$, and is a base-point of $\phi^{-j}$ for $\left.j \geq N\right\}$,

$\mathcal{B}_{+-}=\left\{p \mid p\right.$ is a base-point of $\phi^{j}$, but is not a base-point of $\phi^{-j}$ for $\left.j \geq N\right\}$,

$\mathcal{B}_{-+}=\left\{p \mid p\right.$ is not a base-point of $\phi^{j}$, but is a base-point of $\phi^{-j}$ for $\left.j \geq N\right\}$,

$\mathcal{B}_{--}=\left\{p \mid p\right.$ is not a base-point of $\phi^{j}$, and is not a base-point of $\phi^{-j}$ for $\left.j \geq N\right\}$.

Note that $\mathcal{B}_{+-}$is the set of persistent base-points of $\phi$ and that $\mathcal{B}_{-+}$is the set of persistent base-point of $\phi^{-1}$. This decomposes the set of base-points of $\phi$ into five disjoint sets. Two base-points $p, p^{\prime}$ of $\phi$ which are equivalent belong to the same set.

We fix an integer $k \geq 2 N$ and compute the number of base-points of $\phi^{k}$. Any such base-point being equivalent to a base-point of $\phi$, we take a base-point $p$ of $\phi$, and count the number $m_{p, k}$ of base-points of $\phi^{k}$ which are equivalent to $p$.

If $p$ belongs to $\mathcal{P}$, the number of points equivalent to $p$ is less than $\#(\widehat{\mathcal{P}})$, so $m_{p, k} \leq \#(\widehat{\mathcal{P}})$.

If $p$ is not in $\mathcal{P}$, any point equivalent to $p$ is equal to $\left(\phi^{i}\right)^{\bullet}(p)$ for some $i$, and all are distinct, so we have

$m_{p, k}=\# I_{p, k}$, where

$I_{p, k}=\left\{i \in \mathbb{Z} \mid p\right.$ is not a base-point of $\phi^{i}$, but $p$ is a base-point of $\left.\phi^{i+k}\right\}$.

If $p \in \mathcal{B}_{++}$, since $p$ is not a base-point of $\phi^{i}$ one has $-N<i<N$, thus $m_{p, k}<$ $2 N$.

A point $p \in \mathcal{B}_{--}$is a base-point of $\phi^{i+k}$ hence $-N<i+k<N$ and $m_{p, k}<2 N$.

If $p \in \mathcal{B}_{-+}$, the fact that $p$ is not a base-point of $\phi^{i}$ implies that $-N<i$ and the fact that $p$ is a base-point of $\phi^{i+k}$ implies that $i+k \leq N$. With these two inequalities one has $-N<i \leq N-k$. Since $k>2 N$, we get $m_{p, k}=0$.

If $p \in \mathcal{B}_{+-}$, the fact that $p$ is not a base-point of $\phi^{i}$ implies that $i<N$ and the fact that $p$ is a base-point of $\phi^{i+k}$ implies that $-N<i+k$. This yields $-N-k<i<N$, so $m_{p, k} \leq 2 N+k$. Conversely, if $i \leq-N$ and $i+k \geq N$, $p$ is not a base-point of $\phi^{i}$, but $p$ is a base-point of $\phi^{i+k}$ (or equivalently $i \in I_{p, k}$ ), so $m_{p, k} \geq \#[N-k,-N]=k-2 N+1$. The two conditions together imply that $m_{p, k}-k \in[-2 N, 2 N]$.

Recall that $\mathcal{B}_{+-}$is the set of persistent base-points of $\phi$. The above counting explains that the number of base-points of $\phi^{k}$, for $k$ big, behaves like $v k$, where $v$ is the number of equivalence classes of persistent base-points of $\phi$. More precisely, there exist two integers $c, d$ which do not depend on $k$, such that the total number of base-points of $\phi^{k}$ is between $v k+c$ and $v k+d$, for any $k \geq 2 N$. Recalling that $\mu(\phi)=\lim _{k \rightarrow+\infty} \frac{\mathfrak{b}\left(\phi^{k}\right)}{k}$, where $\mathfrak{b}\left(\phi^{k}\right)$ is the number of base-points of $\phi^{k}$, we obtain $\mu(\phi)=v$. 
Proposition 3.5. Let $\mathrm{S}$ be a smooth projective surface, and let $\phi \in \operatorname{Bir}(\mathrm{S})$. The following conditions are equivalent:

(1) $\mu(\phi)=0$;

(2) $\phi$ is conjugate to an automorphism of a smooth projective surface.

Proof. Lemma 3.1 yields (2) $\Rightarrow$ (1). It suffices then to show (1) $\Rightarrow$ (2).

By blowing-up points, we can always assume that no point of $\mathrm{S}$ is a base-point of $\phi^{k}$ and $\phi^{-l}$ for $k, l>0$; this means that $\phi$ is algebraically stable (see [13]).

Using the notation of Proposition 3.4, this implies that there is no periodic base-point and that $\mathcal{B}_{++}=\emptyset$. Moreover, the condition $\mu(\phi)=0$ corresponds to saying that $\mathcal{B}_{+-}=\mathcal{B}_{-+}=\emptyset$, so all base-points belong to $\mathcal{B}_{--}$.

Suppose that $\phi$ is not an automorphism, and write $\tau: X \rightarrow S$ the blow-up of the base-points of $\phi$, and $\chi: \mathrm{X} \rightarrow \mathrm{S}$ the morphism $\chi=\phi \tau$, which is the blow-up of the base-points of $\phi^{-1}$. Denote by $E \subset \mathrm{X}$ a $(-1)$-curve (smooth rational curve of self-intersection -1 ) contracted by $\chi$. The image $\chi(E)$ is a proper point of $S$, which is a base-point of $\phi^{-1}$. Because $\phi$ is algebraically stable, $\chi(E)$ is not a basepoint of $\phi^{k}$, for any $k \geq 0$. This implies that $\phi^{k} \chi: X \rightarrow S$ is well-defined at any point of $E$, so the curve $C=\tau(E)$ is an irreducible curve of $\mathrm{S}$, contracted by $\phi^{k+1}$, and any base-point of $\phi^{k+1}$ that belongs to $C$, as proper or infinitely near point, is also a base-point of $\phi$. This finite set of points being in $\mathcal{B}_{--}$, there is an integer $n>0$ such that no base-point of $\phi^{n}$ belongs to $C$. Since $C$ is contracted by $\phi^{n}, C$ is a (-1)-curve of $\mathrm{S}$. Contracting $C$ conjugates $\phi$ to an algebraically stable birational map having again all its base-points in $\mathcal{B}_{--}$.

This process decreases the rank of the Picard group of the surfaces obtained; it thus has to stop at some point. This means that $\phi$ is conjugate to an automorphism of a smooth projective surface.

Remark 3.6. In [13, Theorem 0.4] one can find a characterisation of hyperbolic birational maps $\phi$ which are conjugate to an automorphism of a projective surface. If $\phi \in \operatorname{Bir}\left(\mathbb{P}^{2}\right)$ is hyperbolic, we conjugate it to a birational map of a smooth projective surface $\mathrm{S}$ where the action is algebraically stable (this means that $\left(\left.\phi\right|_{\mathrm{H}^{1,1}(\mathrm{~S})}\right)^{n}=\left.\left(\phi^{n}\right)\right|_{\mathrm{H}^{1,1}(\mathrm{~S})}$ for each $\left.n\right)$; its action on $\mathrm{H}^{1,1}(\mathrm{~S})$ admits the eigenvalue $\lambda(\phi)>1$ with eigenvector $\theta_{+}$. The map $\phi$ is birationally conjugate to an automorphism if and only if $\left(\theta_{+}\right)^{2}=0$.

Proposition 3.5 gives another characterisation, for all maps $\phi \in \operatorname{Bir}\left(\mathbb{P}^{2}\right)$ (not only hyperbolic maps), depending only on $\mu(\phi)$.

Example 3.7. In [2-5, 12], automorphisms with positive entropy are constructed starting from a birational map of $\mathbb{P}^{2}$. In most cases, the authors take a birational map $\psi \in \operatorname{Bir}\left(\mathbb{P}^{2}\right)$, and choose $A \in \operatorname{Aut}\left(\mathbb{P}^{2}\right)$ such that $A \psi$ is conjugate to an automorphism of a surface with dynamical degree $>1$. The way to find $A$ is exactly to ensure that $A \psi$ has no persistent base-point (i.e. $\mu(A \psi)=0)$. Let us give an example ([12]):

Let $\phi=A \psi$ be the birational map given by

$$
A:(x: y: z) \rightarrow\left(\alpha x+2(1-\alpha) y+\left(2+\alpha-\alpha^{2}\right) z:-x+(\alpha+1) z: x-2 y+(1-\alpha) z\right)
$$


with $\alpha \in \mathbb{C} \backslash\{0,1\}$ and

$$
\psi:(x: y: z) \rightarrow\left(x z^{2}+y^{3}: y z^{2}: z^{3}\right) .
$$

The map $\psi$ (respectively $\left.\psi^{-1}\right)$ has five base-points, $p=(1: 0: 0)$ and four points infinitely near; we will denote $\widehat{P}_{1}$ (respectively $\widehat{P}_{2}$ ) the collection of these points. The automorphism $A$ is chosen such that:

- $\widehat{P}_{1}, A \widehat{P}_{2}, A \psi A \widehat{P}_{2}$ have distinct supports;

- $\widehat{P}_{1}=(A \psi)^{2} A \widehat{P}_{2}$.

In particular the base-points of $\phi$ are non-persistent, so $\phi$ is conjugate to an automorphism of a rational surface. More precisely $\phi$ is conjugate to an automorphism with positive entropy on $\mathbb{P}^{2}$ blown up in $\widehat{P}_{1}, A \widehat{P}_{2}$ and $A \psi A \widehat{P}_{2}$ (see $[12$, Theorem 3.1]).

\section{Growth of Jonquières twists}

As we explained at the beginning of $\S 1$, Jonquières elements of the Cremona group are those which preserve a pencil of rational curves (which can be sent by a birational transformation of the plane onto a pencil of lines). Among them, those which have a linear degree growth are Jonquières twists, the others are elliptic elements of the Cremona group. This section describes precisely the possible degree growth, and the relation with the pencil of rational curves. Before doing this, let us fix some convention:

\section{Definition 4.1 (Image and preimage of a linear system, free intersection).}

In this article, all linear systems considered are without fixed component (and thus of dimension $\geq 1$ ).

Let $\phi: S \rightarrow S^{\prime}$ be a birational map between two smooth projective surfaces.

Let $\Lambda^{\prime}$ be a linear system of curves on $S^{\prime}$, without fixed component. The preimage of $\Lambda^{\prime}$ by $\phi$, written $\phi^{-1}\left(\Lambda^{\prime}\right)$, is the linear system whose general members are the strict transforms of the general members of $\Lambda^{\prime}$. By construction, $\phi^{-1}\left(\Lambda^{\prime}\right)$ is again a linear system of the same dimension as $\Lambda^{\prime}$ and has no fixed component.

If $\Lambda$ is a linear system of curves on $S$, without fixed component, we similarly define the image of $\Lambda$ by $\phi$, written $\phi(\Lambda)$, so that $\phi^{-1}(\phi(\Lambda))=\Lambda$ and $\phi\left(\phi^{-1}\left(\Lambda^{\prime}\right)\right)=\Lambda^{\prime}$.

This gives in particular an action of $\operatorname{Bir}(S)$ on the set of all linear systems of $S$ having no fixed component.

If $\Lambda_{1}, \Lambda_{2}$ are two linear systems of $S$, the free intersection of $\Lambda_{1}$ and $\Lambda_{2}$ is a non-negative integer, which counts the number of "free points", i.e. points which are not base-points of $\Lambda_{1}, \Lambda_{2}$, in the intersection between a general member of $\Lambda_{1}$ and a general member of $\Lambda_{2}$.

By definition, the free intersection of $\Lambda_{1}$ and $\Lambda_{2}$ is the same as the free intersection of $\phi\left(\Lambda_{1}\right)$ and $\phi\left(\Lambda_{2}\right)$. 
Lemma 4.2. Let $\phi$ be a birational map of $\mathbb{P}^{2}$ which preserves the pencil of lines passing through some point $p_{0}$. The set

$$
\left\{\operatorname{deg} \phi^{k}-k \cdot \frac{\mu(\phi)}{2} \mid k \geq 0\right\} \subset \mathbb{Z}
$$

is bounded.

In particular, the sequence $\left\{\operatorname{deg} \phi^{k}\right\}_{k \in \mathbb{N}}$ grows linearly if and only if $\mu(\phi)>0$ and its growth is given by

$$
\frac{\mu(\phi)}{2} \in \frac{1}{2} \mathbb{N} .
$$

Remark 4.3. Conjugating $\phi$ by a map which preserves the pencil does not change the growth $\left\{\operatorname{deg} \phi^{k}\right\}_{k \in \mathbb{N}}$, but conjugating it by a map which does not preserve the pencil can increase it (see Proposition 4.5).

Proof. Denote by $\Lambda_{0}$ the linear system of lines of $\mathbb{P}^{2}$, and for any integer $k$ we denote by $\Lambda_{k}$ the linear system $\phi^{k}\left(\Lambda_{0}\right)$, which has degree $\operatorname{deg} \phi^{k}=\operatorname{deg} \phi^{-k}$.

For any $k, \phi^{k}$ preserves the pencil of lines passing through $p_{0}$. Computing the free intersection of this pencil with $\Lambda_{k}$, we obtain 1 . This implies that $\Lambda_{k}$ has multiplicity $\operatorname{deg} \phi^{k}-1$ at $p_{0}$, so all other base-points of the pencil have multiplicity 1. Computing the self-intersection, we find that there are exactly $2\left(\operatorname{deg} \phi^{k}-1\right)$ other base-points, all of multiplicity 1 . In particular, $\operatorname{deg} \phi^{k}=\left\lfloor\frac{\mathfrak{b}\left(\phi^{k}\right)}{2}\right\rfloor$. The result follows then directly from Proposition 3.4.

Example 4.4. Let us consider the family of birational maps studied in [11] and defined as

$$
f_{\alpha, \beta}:(x: y: z) \rightarrow((\alpha x+y) z: \beta y(x+z): z(x+z)), \quad \alpha, \beta \in \mathbb{C}^{*} .
$$

Any of the $f_{\alpha, \beta}$ has three base-points: $(1: 0: 0),(0: 1: 0)$ and $(-1: \alpha: 1)$, and preserves the pencil of lines passing through $(1: 0: 0)$. Checking that $(-1:$ $\alpha: 1)$ is the only one persistent base-point ([11, Theorem 1.6]), the growth of $\left\{\operatorname{deg} f_{\alpha, \beta}^{k}\right\}_{k \in \mathbb{N}}$ is given by $\frac{k}{2}$ (see [11, Lemma 1.4]).

Proposition 4.5. Let $\phi \in \operatorname{Bir}\left(\mathbb{P}^{2}\right)$ be a Jonquières twist. There exists an integer $a \in \mathbb{N}$ such that

$$
\lim _{k \rightarrow+\infty} \frac{\operatorname{deg}\left(\phi^{k}\right)}{k}=a^{2} \frac{\mu(\phi)}{2} .
$$

Moreover, $a$ is the degree of the curves of the unique pencil of rational curves invariant by $\phi$. In particular, $a=1$ if and only if $\phi$ preserves a pencil of lines.

Proof. Since $\phi$ is a Jonquières twist, it preserves a unique pencil of rational curves. Taking a birational map $\psi \in \operatorname{Bir}\left(\mathbb{P}^{2}\right)$ which sends this pencil onto a pencil of lines, the birational map $\phi^{\prime}=\psi \phi \psi^{-1}$ preserves the pencil of lines passing through some point $p \in \mathbb{P}^{2}$. Let $\pi: \mathbb{F}_{1} \rightarrow \mathbb{P}^{2}$ be the blow-up of $p \in \mathbb{P}^{2}$, and let $\phi \in \operatorname{Bir}\left(\mathbb{F}_{1}\right)$ be 
defined by $\widehat{\phi}=\pi^{-1} \phi^{\prime} \pi=\pi^{-1} \psi \phi \psi^{-1} \pi$. Denote by $L_{\mathbb{P}^{2}}$ the linear system of lines of $\mathbb{P}^{2}$ and write $\Lambda=\pi^{-1} \psi\left(L_{\mathbb{P}^{2}}\right)$, which is a linear system on $\mathbb{F}_{1}$ (see Definition 4.1 for the precise definition of $\left.\pi^{-1} \psi\left(L_{\mathbb{P}^{2}}\right)\right)$.

The degree of $\phi^{k}$ is equal to the free intersection of $L_{\mathbb{P} 2}$ with $\phi^{k}\left(L_{\mathbb{P}^{2}}\right)$, which is the free intersection of $\Lambda$ with $\widehat{\phi}^{k}(\Lambda)$ (see Definition 4.1).

On $\mathbb{F}_{1}, \Lambda$ is linearly equivalent to $a L+b f$, where $L=\pi^{-1}\left(L_{\mathbb{P}^{2}}\right)$ and $f$ is the divisor of a fibre of $\mathbb{F}_{1} \rightarrow \mathbb{P}^{1}$ and where $a, b \in \mathbb{N}$. Because $\phi^{\prime}$ preserves the pencil of lines through $p, \widehat{\phi}$ preserves the linear system given by $|f|$. To compute $\widehat{\phi}^{k}(\Lambda)$, we want to compute $\hat{\phi}^{k}(L)=\pi^{-1}\left(\phi^{\prime}\right)^{k} \pi(L)=\pi^{-1}\left(\left(\phi^{\prime}\right)^{k}\left(L_{\mathbb{P}^{2}}\right)\right)$. Denoting by $d_{k}$ the degree of $\left(\phi^{\prime}\right)^{k}\left(L_{\mathbb{P}^{2}}\right)$, which is the degree of $\left(\phi^{\prime}\right)^{k}$, the linear system $\left(\phi^{\prime}\right)^{k}\left(L_{\mathbb{P}^{2}}\right)$ has degree $d_{k}$ and multiplicity $d_{k}-1$ at $p$ (see the proof of Lemma 4.2). Hence $\widehat{\phi}^{k}(L)$ is linearly equivalent to $L+\left(d_{k}-1\right) f$, which implies that

$$
\widehat{\phi}^{k}(\Lambda) \sim a L+\left(a\left(d_{k}-1\right)+b\right) f,
$$

so the intersection number of $\widehat{\phi}^{k}(\Lambda)$ with $\Lambda$ is $a^{2} d_{k}+2 a b$. Because $\widehat{\phi}^{k}(\Lambda) \cdot f=a$, each base-point of $\widehat{\phi}^{k}(\Lambda)$ has at most multiplicity $a$. It follows from Lemma 4.2 that $\lim _{k \rightarrow+\infty} \frac{d_{k}}{k}=\frac{\mu(\phi)}{2}$. The number of base-points of $\Lambda$ being bounded, the free intersection of $\widehat{\phi}^{k}(\Lambda)$ with $\Lambda$ grows like $a^{2} \frac{\mu(\phi)}{2} \cdot k$.

Note that $a=f \cdot \Lambda$ is also the free intersection of $\Lambda$ with the linear system $|f|$, since $|f|$ has no base-point. In consequence, $a$ is the free intersection of $\psi^{-1} \pi(\Lambda)=L_{\mathbb{P}^{2}}$ with $\psi^{-1} \pi(|f|)$, which is the degree of the linear system $\psi^{-1} \pi(|f|)$. This gives the result, since $\psi^{-1} \pi(|f|)$ is the unique pencil invariant by $\phi$.

Lemma 4.2 and the second assertion of Lemma 3.1 imply the following statement of [13, Theorem 0.2.]

Corollary 4.6. Let $\phi$ be a Jonquières twist; then $\phi$ is not conjugate to an automorphism.

We can also derive the following new results.

Corollary 4.7. Let $\phi$ be a Jonquières twist. If $\phi^{m}$ and $\phi^{n}$ are conjugate in $\operatorname{Bir}\left(\mathbb{P}^{2}\right)$ for some $m, n \in \mathbb{Z}$, then $|m|=|n|$.

Proof. The fact that $\left\{\operatorname{deg} \phi^{k}\right\}_{k \in \mathbb{N}}$ grows linearly implies that $\phi$ preserves a pencil of rational curves [13, Theorem 0.2]. In particular $\phi$ is conjugate to a birational map of $\mathbb{P}^{2}$ which preserves the pencil of lines passing through some fixed point $p_{0}$. According to Lemma 4.2, one finds $\mu(\phi)>0$.

As $\phi^{m}$ and $\phi^{n}$ are conjugate in $\operatorname{Bir}\left(\mathbb{P}^{2}\right)$ it follows from Lemma 3.1 that $\mu\left(\phi^{m}\right)=\mu\left(\phi^{n}\right)$. Since $\mu\left(\phi^{k}\right)=|k \cdot \mu(\phi)|$ for any $k$, we get $|m|=|n|$. 


\section{Growth of Halphen twists}

In Section 4 (especially Lemma 4.2), we described the degree growth of a Jonquières twist $\phi$, and showed that it is given by $\mu(\phi)$, a birational invariant given by the growth of base-points. For a Halphen twist, the dynamical number of basepoints is trivial, but the growth can also be quantified by an invariant. Recall (see $\S 1$ and references there) that a Halphen twist preserves a unique pencil of elliptic curves, which can be sent by a birational map of the plane onto a Halphen pencil. By [14, Theorem 2 and Proposition 7, page 127], a power of $\phi$ preserves any member of the pencil, and acts on this via a translation.

Proposition 5.1. Let $\phi \in \operatorname{Bir}\left(\mathbb{P}^{2}\right)$ be a Halphen twist.

(1) The set

$$
\left\{\lim _{k \rightarrow+\infty} \frac{\operatorname{deg}\left(\psi \phi^{k} \psi^{-1}\right)}{k^{2}} \mid \psi \in \operatorname{Bir}\left(\mathbb{P}^{2}\right)\right\}
$$

admits a minimum, which is a positive rational number $\kappa(\phi) \in \mathbb{Q}$. If $\phi$ acts via a translation on each member of its invariant pencil of elliptic curves, then $\kappa(\phi) \in 9 \mathbb{N}$.

(2) There exists an integer $a \geq 3$ such that $\lim _{k \rightarrow+\infty} \frac{\operatorname{deg}\left(\phi^{k}\right)}{k^{2}}=\kappa(\phi) \cdot \frac{a^{2}}{9}$.

(3) The following conditions are equivalent:

(a) $a=3$;

(b) $\phi$ preserves a Halphen pencil, i.e. a pencil of (elliptic) curves of degree $3 m$ passing through 9 points with multiplicity $m$.

Remark 5.2. The proof also shows that the degree of the unique pencil of elliptic curves invariant by $\phi$ is equal to $\mathrm{am}$, where $3 \mathrm{~m}$ is the degree of the corresponding Halphen pencil.

Proof. A Halphen twist preserves a unique pencil of elliptic curves. Denoting by $\psi \in \operatorname{Bir}\left(\mathbb{P}^{2}\right)$ an element which sends this pencil onto a Halphen pencil, the birational map $\phi^{\prime}=\psi \phi \psi^{-1}$ preserves a Halphen pencil. Denoting by $\pi: S \rightarrow \mathbb{P}^{2}$ the blow-up of the 9 base-points of the pencil, $\widehat{\phi}=\pi^{-1} \phi^{\prime} \pi$ is an automorphism of $\mathrm{S}$, which preserves the elliptic fibration $\mathrm{S} \rightarrow \mathbb{P}^{1}$ given by $\left|-m K_{\mathrm{S}}\right|$ for some positive integer $m$.

Replacing $\widehat{\phi}$ by some power if needed, we can assume that $\widehat{\phi}$ is a translation on a general fibre. As explained in the proof of [14, Proposition 9, page 132], this yields the existence of an element $\Delta \in \operatorname{Pic}(\mathrm{S})$ (depending on $\widehat{\phi}$ ) with $\Delta \cdot K_{\mathrm{S}}=0$ such that the action of $\widehat{\phi}$ on $\operatorname{Pic}(\mathrm{S})$ is given by

$$
D \mapsto D-m\left(D \cdot K_{\mathrm{S}}\right) \cdot \Delta+\gamma K_{\mathrm{S}},
$$


where $\gamma$ is an integer depending on $D$ which can be computed from the fact that both sides of the equation have the same self-intersection:

$$
\gamma=-\frac{m^{2}}{2}\left(D \cdot K_{\mathrm{S}}\right) \cdot \Delta^{2}+m(D \cdot \Delta)
$$

We denote by $L$ the linear system of lines of $\mathbb{P}^{2}$ and by $\Lambda=\pi^{-1} \psi(L)$ the corresponding system on S (see Definition 4.1 for the precise meaning of $\pi^{-1} \psi(L)$ ). The degree of $\phi^{n}$ is equal to the free intersection of $L$ with $\phi^{n}(L)$, which is equal to the free intersection of $\Lambda$ with $\widehat{\phi}^{n}(\Lambda)$ (as explained in Definition 4.1).

The map $\widehat{\phi}^{n}$ acts on $\operatorname{Pic}(\mathrm{S})$ as

$$
D \mapsto D-m\left(D \cdot K_{\mathrm{S}}\right) \cdot(n \Delta)+\left(-\frac{m^{2}}{2}\left(D \cdot K_{\mathrm{S}}\right) \cdot(n \Delta)^{2}+m(D \cdot(n \Delta))\right) K_{\mathrm{S}}
$$

This yields

$$
\begin{aligned}
\Lambda \cdot \widehat{\phi}^{n}(\Lambda)= & \Lambda^{2}-m\left(\Lambda \cdot K_{\mathrm{S}}\right) \cdot(n \Delta \cdot \Lambda) \\
& +\left(-\frac{m^{2}}{2}\left(\Lambda \cdot K_{\mathrm{S}}\right)(n \Delta)^{2}+m(n \Lambda \cdot \Delta)\right)\left(K_{\mathrm{S}} \cdot \Lambda\right) \\
= & \Lambda^{2}+\left(-\frac{m^{2}}{2}\left(\Lambda \cdot K_{\mathrm{S}}\right)^{2}(\Delta)^{2}\right) \cdot n^{2} .
\end{aligned}
$$

The free intersection between $\Lambda$ and $\widehat{\phi}^{n}(\Lambda)$ is thus equal to

$$
\Lambda^{2}+\left(-\frac{m^{2}}{2}\left(\Lambda \cdot K_{\mathrm{S}}\right)^{2}(\Delta)^{2}\right) \cdot n^{2}-\sum_{i=1}^{k} \mu_{i}(\Lambda) \cdot \mu_{i}\left(\widehat{\phi}^{n}(\Lambda)\right)
$$

where $\mu_{i}(\Lambda)$ and $\mu_{i}\left(\widehat{\phi}^{n}(\Lambda)\right)$ denote the multiplicities of respectively $\Lambda$ and $\widehat{\phi}^{n}(\Lambda)$ at the $r$ base-points of $\Lambda$. Since $\widehat{\phi}$ is an automorphism of S, the contribution given by the base-points is bounded, so we find that

$$
\lim _{n \rightarrow+\infty} \frac{\operatorname{deg}\left(\phi^{n}\right)}{n^{2}}=m^{2}\left(\Lambda \cdot K_{\mathrm{S}}\right)^{2} \cdot\left(\frac{-\Delta^{2}}{2}\right) .
$$

Note that $\Lambda$ is the lift by $\pi^{-1}$ of the homaloidal linear system $\psi(L)$, so $\Lambda \cdot\left(-K_{\mathrm{S}}\right) \geq$ 3 , and equality holds if and only if $\Lambda$ has no base-point. This shows that the minimum among all homaloidal systems is attained when $\Lambda$ has no base-point; we get $\kappa(\phi)=9 m^{2} \cdot\left(\frac{-\Delta^{2}}{2}\right)$ and $a=\Lambda \cdot K_{\mathrm{S}}$.

Let us prove that $-\Delta^{2}$ is a positive even number. To do this, we take the orthogonal basis $L, E_{1}, \ldots, E_{9}$ of $\operatorname{Pic}(\mathrm{S})$, where $L$ is the pull-back of a line of $\mathbb{P}^{2}$ and $E_{1}, \ldots, E_{9}$ are the exceptional divisors associated to the points blown-up. Writing

$$
\Delta=d L-\sum_{i=1}^{9} a_{i} E_{i}
$$


the equality $\Delta \cdot K_{\mathrm{S}}=0$ implies that $\sum a_{i}=3 d$. Modulo 2 , we have $\sum\left(a_{i}\right)^{2} \equiv$ $\sum a_{i} \equiv 3 d \equiv d^{2}$, so $\Delta^{2}=d^{2}-\sum\left(a_{i}\right)^{2}$ is even. Applying Cauchy-Schwarz to $(1, \ldots, 1)$ and $\left(a_{1}, \ldots, a_{9}\right)$, we get $9 \sum\left(a_{i}\right)^{2} \geq\left(\sum a_{i}\right)^{2}$, and equality holds only when all $a_{i}$ are equal. This latter would imply that $\Delta$ is a multiple of $K_{\mathrm{S}}$, and thus that $\widehat{\phi}$ acts trivially on $\operatorname{Pic}(\mathrm{S})$. Hence we have $9 \sum\left(a_{i}\right)^{2}>\left(\sum a_{i}\right)^{2}=(3 d)^{2}$, which implies that $-\Delta^{2}=\sum\left(a_{i}\right)^{2}-d^{2}>0$.

The fact that $-\Delta^{2}$ is a positive even number implies that $\kappa(\phi)=9 m^{2} \cdot\left(\frac{-\Delta^{2}}{2}\right)$ is a positive integer divisible by 9 . The equality $\kappa(\phi)=\lim _{k \rightarrow+\infty} \frac{\operatorname{deg}\left(\phi^{k}\right)}{k^{2}}$ is equivalent to the fact that $\Lambda$ has no base-point, which corresponds to say that $\psi^{-1} \pi$ is a birational morphism, or equivalently that the unique pencil of elliptic curves invariant by $\phi$ is a Halphen pencil.

Corollary 5.3. Let $\phi \in \operatorname{Bir}\left(\mathbb{P}^{2}\right)$ be a Halphen twist. The integer $\kappa(\phi)$ is a birational invariant which satisfies $\kappa\left(\phi^{m}\right)=m^{2} \kappa(\phi)$ for any $m \in \mathbb{Z}$. In particular, the maps $\phi^{n}$ and $\phi^{m}$ are not conjugate if $|m| \neq|n|$.

Proof. Is a direct consequence of Proposition 5.1.

Let us give an example where $\kappa(\phi)$ is not an integer.

Example 5.4. Let $\Lambda$ be the pencil of cubic curves of $\mathbb{P}^{2}$ given by $\lambda\left(x^{2} y+z^{3}+\right.$ $\left.y^{2} z\right)+\mu\left(x^{2} z+y^{3}+y z^{2}\right)=0,(\lambda: \mu) \in \mathbb{P}^{1}$. This pencil is invariant by the automorphism $\alpha \in \operatorname{Aut}\left(\mathbb{P}^{2}\right)$ defined by $(x: y: z) \mapsto(\mathbf{i} x:-y: z)$.

Denote by $\pi: S \rightarrow \mathbb{P}^{2}$ the blow-up of the base-points of $\Lambda$, which are 7 proper points of $\mathbb{P}^{2}$ and 2 infinitely near points. More precisely, the 7 proper points are

$$
\begin{aligned}
& p_{1}=(1: 0: 0), \\
& p_{2}=(\mathbf{i} \sqrt{2}: 1: 1), \\
& p_{3}=\alpha\left(p_{2}\right)=(-\sqrt{2}:-1: 1), \\
& p_{4}=\alpha\left(p_{3}\right)=(-\mathbf{i} \sqrt{2}: 1: 1), \\
& p_{5}=\alpha\left(p_{4}\right)=(\sqrt{2}:-1: 1), \\
& p_{6}=(0: \mathbf{i}: 1), \\
& p_{7}=\alpha\left(p_{6}\right)=(0:-\mathbf{i}: 1) .
\end{aligned}
$$

The last two points are the following: the point $p_{8}$ is infinitely near to $p_{6}$, corresponding to the tangent direction of the line $y=\mathbf{i} z$, and $p_{9}$ is infinitely near to $p_{7}$, corresponding to the tangent direction of the line $y=-\mathbf{i} z$.

The surface $\mathrm{S}$ inherits an elliptic fibration $\mathrm{S} \rightarrow \mathbb{P}^{1}$, and the lift of $\alpha$ yields an automorphism $\widehat{\alpha}=\pi \alpha \pi^{-1}$ of S. Denote by $E_{i} \in \operatorname{Pic}(\mathrm{S})$ the divisor of selfintersection -1 corresponding to the point $p_{i}$. If $i \neq 6,7$, then $E_{i}$ corresponds to a $(-1)$-curve of $\mathrm{S}$; and $E_{6}, E_{7}$ correspond to two reducible curves of $\mathrm{S}$. 
For any $\Delta \in \operatorname{Pic}(\mathrm{S})$ satisfying $\Delta \cdot K_{\mathrm{S}}=0$, we denote by $\iota_{\Delta} \in \operatorname{Aut}(\mathrm{S})$ the automorphism which restricts on a general fibre $C$ to the translation given by the divisor $\left.\Delta\right|_{C}$. If $\Delta^{2}=-2$, the action of $\iota_{\Delta}$ on $\operatorname{Pic}(\mathrm{S})$ is given by (see the proof of Proposition 5.1)

$$
D \mapsto D-\left(D \cdot K_{\mathrm{S}}\right) \cdot \Delta+\left(D \cdot\left(K_{\mathrm{S}}+\Delta\right)\right) \cdot K_{\mathrm{S}}
$$

For any automorphism $\sigma \in \operatorname{Aut}(\mathrm{S})$, one can check that $\iota_{\sigma(\Delta)}=\sigma \iota_{\Delta} \sigma^{-1}$. In particular, we have

$$
\begin{aligned}
\left(\widehat{\alpha} \iota_{\Delta}\right)^{4} & =\left(\widehat{\alpha} \iota_{\Delta} \widehat{\alpha}^{-1}\right)\left(\widehat{\alpha}^{2} \iota_{\Delta} \widehat{\alpha}^{-2}\right)\left(\widehat{\alpha}^{3} \iota_{\Delta} \widehat{\alpha}^{-3}\right) \iota_{\Delta} \\
& =\iota_{\widehat{\alpha}(\Delta)} \iota_{\widehat{\alpha}^{2}(\Delta)} \iota_{\widehat{\alpha}^{3}(\Delta)} \iota_{\Delta}=\iota_{\widehat{\alpha}(\Delta)+\widehat{\alpha}^{2}(\Delta)+\widehat{\alpha}^{3}(\Delta)+\Delta .}
\end{aligned}
$$

Because of the action of $\alpha$ on the points $p_{i}$, we have

$$
\begin{array}{ll}
\widehat{\alpha}\left(E_{6}\right)=E_{7}, & \widehat{\alpha}\left(E_{7}\right)=E_{6}, \\
\widehat{\alpha}\left(E_{2}\right)=E_{3}, & \widehat{\alpha}\left(E_{3}\right)=E_{4}, \\
\widehat{\alpha}\left(E_{4}\right)=E_{5}, & \widehat{\alpha}\left(E_{5}\right)=E_{2} .
\end{array}
$$

We now fix $\Delta \in \operatorname{Pic}(\mathrm{S})$ to be the divisor $E_{2}-E_{6}$ (that satisfies $\Delta \cdot K_{\mathrm{S}}=0$ and $\Delta^{2}=-2$ ), and obtain

$$
\widehat{\alpha}(\Delta)+\widehat{\alpha}^{2}(\Delta)+\widehat{\alpha}^{3}(\Delta)+\Delta=E_{2}+E_{3}+E_{4}+E_{5}-2 E_{6}-2 E_{7},
$$

which has square -8 . In particular, the number $\kappa$ associated to

$$
\left(\widehat{\alpha} \iota_{\Delta}\right)^{4}=\iota_{\widehat{\alpha}(\Delta)+\widehat{\alpha}^{2}(\Delta)+\widehat{\alpha}^{3}(\Delta)+\Delta}
$$

is

$$
-9 \frac{\left(\iota_{\widehat{\alpha}(\Delta)+\widehat{\alpha}^{2}(\Delta)+\widehat{\alpha}^{3}(\Delta)+\Delta}\right)^{2}}{2}=36 .
$$

This shows, by Corollary 5.3, that $\kappa(\phi)=\frac{9}{4}$, where $\phi$ is the birational map of $\mathbb{P}^{2}$ conjugate to $\widehat{\alpha} \iota_{\Delta}$ by $\pi^{-1}$, namely $\alpha \pi \iota_{\Delta} \pi^{-1}$.

\section{Applications}

\subsection{Birational maps having two conjugate iterates}

Lemma 6.1. Let $\phi$ denote a birational map of $\mathbb{P}^{2}$. Assume that $\phi^{n}$ and $\phi^{m}$ are conjugate and assume that $|m| \neq|n|$. Then, $\phi$ is elliptic and satisfies $\lambda(\phi)=1$ and $\mu(\phi)=0$. 
Proof. The map $\phi^{m}$ is conjugate to $\phi^{n}$ in $\operatorname{Bir}\left(\mathbb{P}^{2}\right)$ so one gets $\lambda(\phi)^{|m|}=\lambda(\phi)^{|n|}$ and $|m| \cdot \mu(\phi)=|n| \cdot \mu(\phi)$. This yields $\lambda(\phi)=1$ and $\mu(\phi)=0$.

The fact that $\lambda(\phi)=1$ implies that $\phi$ is elliptic, or a Jonquières or Halphen twist. The Jonquières and Halphen cases are impossible (Corollaries 4.7 and 5.3).

Proposition 6.2. Let $\phi$ denote a birational map of $\mathbb{P}^{2}$ of infinite order. Assume that $\phi^{n}$ and $\phi^{m}$ are conjugate and assume that $|m| \neq|n|$. Then, $\phi$ is conjugate to an automorphism of $\mathbb{C}^{2}$ of the form $(x, y) \mapsto(\alpha x, y+1)$, where $\alpha \in \mathbb{C}^{*}$ such that $\alpha^{m+n}=1$ or $\alpha^{m-n}=1$.

In particular, if $\phi$ is conjugate to $\phi^{n}$ for any positive integer $n$, then $\phi$ is conjugate to $(x, y) \mapsto(x, y+1)$.

Proof. This statement follows from Corollary 2.5, Corollary 5.3 and Lemma 6.1.

\subsection{Morphisms of Baumslag-Solitar groups in the Cremona group}

For any integers $m, n$ such that $m n \neq 0$, the $\operatorname{Baumslag-\operatorname {Solitar}group} \operatorname{BS}(m, n)$ is defined by the following presentation

$$
\mathrm{BS}(m, n)=\left\langle r, s \mid r s^{m} r^{-1}=s^{n}\right\rangle .
$$

Recall that if $\mathrm{G}$ is a group, the derived groups of $\mathrm{G}$ are

$$
\mathrm{G}^{(0)}=\mathrm{G}, \quad \mathrm{G}^{(i)}=\left[\mathrm{G}^{(i-1)}, \mathrm{G}^{(i-1)}\right]=\left\langle g h g^{-1} h^{-1} \mid g, h \in \mathrm{G}^{(i-1)}\right\rangle \text { for all } i \geq 1,
$$

and that $\mathrm{G}$ is solvable if there exists an integer $N$ such that $\mathrm{G}^{(N)}=\{\mathrm{id}\}$.

The group $\mathrm{BS}(m, n)$ (respectively the subgroups of finite index of $\operatorname{BS}(m, n))$ is solvable if and only if $|m|=1$ or $|n|=1$ (see [20, Proposition A.6]).

A group $\mathrm{G}$ is said to be residually finite if for any $g$ in $\mathrm{G} \backslash\{\mathrm{id}\}$ there exist a finite group $\mathrm{H}$ and a group homomorphism $\Theta: \mathrm{G} \rightarrow \mathrm{H}$ such that $\Theta(g)$ belongs to $\mathrm{H} \backslash\{\mathrm{id}\}$. The group $\operatorname{BS}(m, n)$ is residually finite if and only if $|m|=1$ or $|n|=1$ or $|m|=|n|$ (see [19]). Let $V$ be an affine algebraic variety; according to [1] any subgroup of finite index of the automorphisms group $\operatorname{Aut}(V)$ of $V$ is residually finite. Therefore if $|m| \neq|n|$ and $|m|,|n| \neq 1$ there is no embedding of $\operatorname{BS}(m, n)$ into the group of polynomial automorphisms of the plane. There is an other proof using the amalgated structure of the group of polynomial automorphisms of the plane and the fact that $\operatorname{BS}(m, n)$ is not solvable ([10, Proposition 2.2]).

Lemma 6.3. Let $\rho$ be a homomorphism from $\mathrm{BS}(m, n)=\left\langle r, s \mid r s^{m} r^{-1}=s^{n}\right\rangle$ to $\operatorname{Bir}\left(\mathbb{P}^{2}\right)$. Assume that $|m|,|n|$ and 1 are distinct. If $\rho(s)$ has infinite order, the image of the subgroup of finite index $\left\langle r, s^{m} \mid r s^{m^{2}} r^{-1}=s^{m n}\right\rangle$ of $\mathrm{BS}(m, n)$ is solvable. 
Proof. By Proposition 6.2, one can conjugate $\rho$ so that $\rho(s):(x, y)--\rightarrow(\alpha x, y+1)$ where $\alpha \in \mathbb{C}^{*}$ and $\alpha^{m-n}=1$ or $\alpha^{m+n}=1$. Denoting respectively by $\psi$ the map $\left(x, \frac{n}{m} y\right)$ or $\left(x^{-1}, \frac{n}{m} y\right)$ one has $\psi \rho(s)^{m} \psi^{-1}=\rho(s)^{n}$. So $\rho(r)=\psi \tau$ where $\tau$ commutes with $\rho(s)^{m}:(x, y) \mapsto\left(\alpha^{m} x, y+m\right)$.

According to Lemma 2.8, one then has $\tau=(\eta(x), y+R(x))$ for some $\eta \in$ PGL $(2, \mathbb{C}), \eta\left(\alpha^{m} x\right)=\alpha^{m} \eta(x)$, and some $R \in \mathbb{C}(x)$ satisfying $R\left(\alpha^{m} x\right)=R(x)$. And one gets $\rho(r)=\left(\eta(x)^{ \pm 1}, \frac{n}{m}(y+R(x))\right)$.

The group generated by $\rho\left(s^{m}\right)$ and $\rho(r)$ is thus solvable.

Corollary 6.4. If $|m|,|n|$ and 1 are distinct, there is no embedding of $\operatorname{BS}(m, n)$ into the Cremona group.

Proof. Lemma 6.3 shows that the image of any embedding would be virtually solvable, impossible when $|m|,|n|$ and 1 are distinct.

\subsection{Embeddings of $\mathrm{GL}(2, \mathbb{Q})$ into the Cremona group}

To simplify the notation, we will denote in this last section by $\left(\phi_{1}(x, y), \phi_{2}(x, y)\right)$ the rational map $(x, y) \rightarrow\left(\phi_{1}(x, y), \phi_{2}(x, y)\right)$ from $\mathbb{C}^{2}$ to $\mathbb{C}^{2}$.

Let us first give examples of embeddings of $\mathrm{GL}(2, \mathbb{Q})$ into the Cremona group.

Examples 6.5. Let $k$ be an odd integer and let $\chi: \mathbb{Q}^{*} \rightarrow \mathbb{C}^{*}$ be a homomorphism such that $a \mapsto \frac{\chi\left(a^{2}\right)}{a^{k}}$ is injective. The morphism $\rho$ from $\operatorname{GL}(2, \mathbb{Q})$ to the Cremona group given by

$$
\rho\left(\left[\begin{array}{ll}
a & b \\
c & d
\end{array}\right]\right)=\left(x \cdot \frac{\chi(a d-b c)}{(c y+d)^{k}}, \frac{a y+b}{c y+d}\right)
$$

is an embedding. Note that $\rho(\mathrm{GL}(2, \mathbb{Q}))$ is conjugate to a subgroup of automorphisms of the $k$-th Hirzebruch surface $\mathbb{F}_{k}$. Changing $k$ gives then infinitely many non conjugate embeddings in the Cremona group.

Remark 6.6. Taking $k=1$ and $\chi$ the trivial map, Example 6.5 yields the embed$\operatorname{ding} \rho: \operatorname{GL}(2, \mathbb{Q}) \rightarrow \operatorname{Bir}\left(\mathbb{P}^{2}\right)$ given by

$$
\rho\left(\left[\begin{array}{ll}
a & b \\
c & d
\end{array}\right]\right)=\left(\frac{x}{c y+d}, \frac{a y+b}{c y+d}\right),
$$

which is obviously conjugate (by extending the actions to $\mathbb{P}^{2}$ ) to the classical embedding

$$
\widehat{\rho}\left(\left[\begin{array}{ll}
a & b \\
c & d
\end{array}\right]\right)=(a x+b y, c x+d y) .
$$

Theorem 6.7. Let $\rho: \mathrm{GL}(2, \mathbb{Q}) \rightarrow \operatorname{Bir}\left(\mathbb{P}^{2}\right)$ be an embedding of $\mathrm{GL}(2, \mathbb{Q})$ into the Cremona group, then up to conjugation $\rho$ is one of the embeddings described in Examples 6.5. 
Proof. Let us set

$$
\mathrm{t}_{q}=\left[\begin{array}{ll}
1 & q \\
0 & 1
\end{array}\right] \quad \& \quad \mathrm{~d}_{m, n}=\left[\begin{array}{cc}
m & 0 \\
0 & n
\end{array}\right], \quad q, m, n \in \mathbb{Q} .
$$

Remark that $\mathrm{t}_{1}$ is conjugate to $\mathrm{t}_{n}$ in $\operatorname{GL}(2, \mathbb{Q})$, for any $n \in \mathbb{Z} \backslash\{0\}$; we can then assume, after conjugation, that $\rho\left(\mathrm{t}_{1}\right)=(x, y+1)$ (Proposition 6.2). As $\rho\left(\mathrm{t}_{1 / n}\right)$ commutes with $\rho\left(\mathrm{t}_{1}\right)$ there exist $A_{n}$ in $\operatorname{PGL}(2, \mathbb{C})$ and $R_{n}$ in $\mathbb{C}(x)$ such that

$$
\rho\left(\mathrm{t}_{1 / n}\right)=\left(A_{n}(x), y+R_{n}(x)\right)
$$

(see Lemma 2.8). Let us prove now that $A_{n}(x)=x$. Since $\mathrm{t}_{1 / n}^{n}=\mathrm{t}_{1}$ the element $A_{n}$ is of finite order so $A_{n}$ is conjugate to some $\xi x$ where $\xi$ is some root of unity. Hence $\rho\left(\mathrm{t}_{1 / n}\right)$ is conjugate to $(\xi x, y+Q(x))$ where $Q \in \mathbb{C}(x)$ satisfies

$$
Q(x)+Q(\xi x)+\cdots+Q\left(\xi^{n-1} x\right)=1 .
$$

The map $(\xi x, y+Q(x))$ is then conjugate to $\left(\xi x, y+\frac{1}{n}\right)$ by $\left(x, y-\frac{\sum_{i=1}^{n-1} i Q\left(\xi^{i} x\right)}{n}\right)$. Since $\mathrm{t}_{1 / n}$ is conjugate to $\mathrm{t}_{1}$, Proposition 2.4 implies that $\xi=1$, which achieves to show that $A_{n}(x)=x$. This implies, with equality $\rho\left(\mathrm{t}_{1 / n}\right)^{n}=\rho\left(\mathrm{t}_{1}\right)$, that $R_{n}(x)=$ $1 / n$. We thus have for any $q$ in $\mathbb{Q}$

$$
\rho\left(\mathrm{t}_{q}\right)=(x, y+q) .
$$

From $\mathrm{d}_{m, n} \mathrm{t}_{1} \mathrm{~d}_{m, n}^{-1}=\mathrm{t}_{m / n}$ one gets (using again Lemma 2.8 ) that

$$
\rho\left(\mathrm{d}_{m, n}\right)=\left(\eta_{m, n}(x), \frac{m}{n} y+R_{m, n}(x)\right), \quad \eta_{m, n} \in \operatorname{PGL}(2, \mathbb{C}), R_{m, n} \in \mathbb{C}(x) .
$$

The map $\left(\mathbb{Q}^{*}\right)^{2} \rightarrow \operatorname{PGL}(2, \mathbb{C})$ given by $(m, n) \mapsto \eta_{m, n}$ is a homomorphism, which cannot be injective. There exists thus one element $\mathrm{d}_{m, n}$ with $(m, n) \neq(1,1)$ such that $\rho\left(\mathrm{d}_{m, n}\right)=\left(x, \frac{m}{n} y+R_{m, n}(x)\right)$. Note that $m \neq n$ since the centralisers of $\rho\left(\mathrm{d}_{m, n}\right)$ and $\rho\left(\mathrm{t}_{1}\right)$ are different. Conjugating by $\left(x, y+\frac{R_{m, n}(x)}{m / n-1}\right)$, we can assume that $\rho\left(\mathrm{d}_{m, n}\right)=\left(x, \frac{m}{n} y\right)$. From $\mathrm{d}_{m, n} \mathrm{~d}_{a, b}=\mathrm{d}_{a, b} \mathrm{~d}_{m, n}$ one gets for any $a, b$ in $\mathbb{Q}$

$$
\rho\left(\mathrm{d}_{a, b}\right)=\left(\eta_{a, b}(x), \frac{a}{b} y\right), \quad \eta_{a, b} \in \operatorname{PGL}(2, \mathbb{C}) .
$$

The homomorphism $\mathbb{Q}^{*} \rightarrow \operatorname{PGL}(2, \mathbb{C})$ given by $a \mapsto \eta_{a, a}$ is injective, so up to conjugation by an element of $\operatorname{PGL}(2, \mathbb{C})$ we can assume that for any $a \in \mathbb{Q} \backslash\{0,1\}$ there exists $\chi_{a, a} \in \mathbb{C} \backslash\{0,1\}$ such that $\eta_{a, a}(x)=\chi_{a, a} x$. This implies the existence of $\chi_{a, b} \in \mathbb{C}^{*}$ for any $a, b$ in $\left(\mathbb{Q}^{*}\right)^{2}$, such that $\eta_{a, b}(x)=\chi_{a, b} x$.

We now compute the image of $M=\left[\begin{array}{cc}0 & 1 \\ -1 & 0\end{array}\right]$. Since $\rho(M)$ commutes with $\rho\left(\mathrm{d}_{2,2}\right)=\left(\chi_{2,2} x, y\right)$ where $\chi_{2,2} \in \mathbb{C}^{*}$ is of infinite order, there exist $R \in \mathbb{C}(y)$ and 
$v \in \operatorname{PGL}(2, \mathbb{C})$ such that $\rho(M)=(x R(y), v(y))$ (Lemma 2.8). For any $a \in \mathbb{Q}^{*}$, equality $M \mathrm{~d}_{a, 1}=\mathrm{d}_{1, a} M$ yields

$$
\left(\chi_{a, 1} x \cdot R(a y), v(a y)\right)=\left(\chi_{1, a} x \cdot R(y), \frac{1}{a} v(y)\right) .
$$

This implies that $R(y)=\alpha y^{-k}$ and $v(y)=\frac{\beta}{y}$, for some $\alpha, \beta \in \mathbb{C}^{*}, k \in \mathbb{Z}$, i.e. $\rho(M)=\left(\alpha \frac{x}{y^{k}}, \frac{\beta}{y}\right)$. We use now equality $\left(M \mathrm{t}_{1}\right)^{3}=\mathrm{id}$ : the second component of $\left(\rho(M) \rho\left(\mathrm{t}_{1}\right)\right)^{3}$ being

$$
\frac{\beta(y+\beta+1)}{(\beta+1) y+2 \beta+1},
$$

we find $\beta=-1$ and compute $\left(\rho(M) \rho\left(\mathrm{t}_{1}\right)\right)^{3}=\left(x \alpha^{3}(-1)^{k}, y\right)$ so $\alpha^{3}=(-1)^{k}$. Since $\rho(M)^{2}=\left(x \alpha^{2}(-1)^{k}, y\right)$ has order 2 , we have $-\alpha^{2}=(-1)^{k}$, thus $\alpha=-1$ and $k$ is odd.

Writing $\chi(a)=\chi a, 1$ for any $a \in \mathbb{Q}$, the map $\mathbb{Q}^{*} \rightarrow \mathbb{C}^{*}$ given by $a \rightarrow \chi(a)$ is a homomorphism, and one gets $\rho\left(\mathrm{d}_{a, 1}\right)=(\chi(a) x, a y)$. The group GL(2, $\left.\mathbb{Q}\right)$ is generated by the maps $\mathrm{d}_{a, 1}, \mathrm{t}_{a}$ and $M$, so

$$
\rho\left(\left[\begin{array}{ll}
a & b \\
c & d
\end{array}\right]\right)=\left(x \cdot \frac{\chi(a d-b c)}{(c y+d)^{k}}, \frac{a y+b}{c y+d}\right)
$$

for any $\left[\begin{array}{ll}a & b \\ c & d\end{array}\right] \in \operatorname{GL}(2, \mathbb{Q})$. This yields an embedding if and only if the homomorphism from $\mathbb{Q}^{*}$ to $\mathbb{C}^{*}$ given by $a \mapsto \frac{\chi\left(a^{2}\right)}{a^{k}}$ is injective.

It remains to observe that $k$ can be chosen to be positive. Indeed, otherwise one conjugates by $\left(\frac{1}{x}, y\right)$ and replaces $\chi$ with $\frac{1}{\chi}$ to replace $k$ with $-k$.

One can see that $\operatorname{GL}(n, \mathbb{Q})$ does not embedd into $\operatorname{Bir}\left(\mathbb{P}^{2}\right)$ as soon as $n \geq 3$. Indeed, Theorem 6.7 implies that the diagonal matrices are sent onto diagonal elements of $\operatorname{PGL}(3, \mathbb{C})=\operatorname{Aut}\left(\mathbb{P}^{2}\right)$, which is impossible, by considering the involutions. One can also find another less obvious corollary:

Corollary 6.8. Let $\rho: \mathrm{GL}(2, \mathbb{C}) \rightarrow \operatorname{Bir}\left(\mathbb{P}^{2}\right)$ be an embedding of $\mathrm{GL}(2, \mathbb{C})$ into the Cremona group. There exist a positive odd integer $k$, a field homomorphism $\tau: \mathbb{C} \rightarrow \mathbb{C}$ and a group homomorphism $\chi: \mathbb{C}^{*} \rightarrow \mathbb{C}^{*}$ such that

$$
\rho\left(\left[\begin{array}{ll}
a & b \\
c & d
\end{array}\right]\right)=\left(x \cdot \frac{\chi(a d-b c)}{(\tau(c) y+\tau(d))^{k}}, \frac{\tau(a) y+\tau(b)}{\tau(c) y+\tau(d)}\right), \quad \forall\left[\begin{array}{ll}
a & b \\
c & d
\end{array}\right] \in \mathrm{GL}(2, \mathbb{C}) .
$$

Remark 6.9. One sees that in the description above, $\rho$ is an embedding if and only if the group homomorphism $\mathbb{C}^{*} \rightarrow \mathbb{C}^{*}$ given by $a \mapsto \frac{\chi\left(a^{2}\right)}{\tau(a)^{k}}$ is injective. This happens for instance by taking $\chi(a)=\tau(a)^{\frac{k \pm 1}{2}}$, any positive odd integer $k$ and any field homomorphism $\tau: \mathbb{C} \rightarrow \mathbb{C}$. 
Proof. The map $\rho$ induces an embedding of $\mathrm{GL}(2, \mathbb{Q})$ into $\operatorname{Bir}\left(\mathbb{P}^{2}\right)$. According to Theorem 6.7 one has a description of $\rho_{\mid \mathrm{GL}(2, \mathbb{Q})}$. Up to conjugacy, there exists an odd positive integer $k$ and an homomorphism $\widetilde{\chi}: \mathbb{Q}^{*} \rightarrow \mathbb{C}^{*}$ such that

$$
\rho\left(\left[\begin{array}{ll}
a & b \\
c & d
\end{array}\right]\right)=\left(x \cdot \frac{\tilde{\chi}(a d-b c)}{(c y+d)^{k}}, \frac{a y+b}{c y+d}\right), \quad \forall\left[\begin{array}{ll}
a & b \\
c & d
\end{array}\right] \in \mathrm{GL}(2, \mathbb{Q}) .
$$

Let us set

$$
\mathrm{t}_{a}=\left[\begin{array}{ll}
1 & a \\
0 & 1
\end{array}\right] \quad \& \quad \mathrm{~d}_{b}=\left[\begin{array}{ll}
b & 0 \\
0 & 1
\end{array}\right], \quad a \in \mathbb{C}, b \in \mathbb{C}^{*} .
$$

For any $a \in \mathbb{C}^{*}$, the matrix $\mathrm{d}_{a}$ commutes with all diagonal matrices with entries in $\mathbb{Q}$; this implies, with the description above, that

$$
\rho\left(\mathrm{d}_{a}\right)=(\chi(a) x, \tau(a) y)
$$

for some $\chi(a), \tau(a)$ in $\mathbb{C}^{*}$ (Lemma 2.7). This yields two group homomorphisms $\chi, \tau: \mathbb{C}^{*} \rightarrow \mathbb{C}^{*}$. Observe that $\chi$ is an extension of $\tilde{\chi}$, i.e. $\chi(a)=\tilde{\chi}(a)$ for any $a \in \mathbb{Q}$.

The equality $\mathrm{d}_{a} \mathrm{t}_{1} \mathrm{~d}_{a^{-1}}=\mathrm{t}_{a}$ implies that

$$
\rho\left(\mathrm{t}_{a}\right)=(x, y+\tau(a)), \quad \forall a \in \mathbb{C}^{*} .
$$

In particular, $\tau$ extends to an (injective) field homomorphism $\mathbb{C} \rightarrow \mathbb{C}$. The group $\operatorname{GL}(2, \mathbb{C})$ being generated by $\operatorname{GL}(2, \mathbb{Q})$ and $\left\{\mathrm{d}_{a} \mid a \in \mathbb{C}^{*}\right\}$, one has

$$
\rho\left(\left[\begin{array}{ll}
a & b \\
c & d
\end{array}\right]\right)=\left(x \cdot \frac{\chi(a d-b c)}{(\tau(c) y+\tau(d))^{k}}, \frac{\tau(a) y+\tau(b)}{\tau(c) y+\tau(d)}\right), \quad \forall\left[\begin{array}{ll}
a & b \\
c & d
\end{array}\right] \in \mathrm{GL}(2, \mathbb{C}) .
$$

The map $\rho$ is injective if and only if $\chi\left(a^{2}\right) \neq \tau\left(a^{k}\right)$ for any $a \in \mathbb{C}^{*} \backslash\{1\}$.

\section{References}

[1] H. BASS and A. LUBOTZKY, Automorphisms of groups and of schemes of finite type, Israel J. Math. 44 (1983), 1-22.

[2] E. BEDFORD and K. KIM, Periodicities in linear fractional recurrences: degree growth of birational surface maps, Michigan Math. J. 54 (2006), 647-670.

[3] E. BEDFORD and K. KIM, Dynamics of rational surface automorphisms: linear fractional recurrences, J. Geom. Anal. 19 (2009), 553-583.

[4] E. BEDFORD and K. KIM, Continuous Families of Rational Surface Automorphisms with Positive Entropy, Math. Ann. 348 (2010), 667-688.

[5] E. BEDFORD and K. KIM, Dynamics of rational surface automorphisms: rotation domains, Amer. J. Math. 134 (2012), 379-405.

[6] J. BLANC, Conjugacy classes of affine automorphisms of $\mathbb{K}^{n}$ and linear automorphisms of $\mathbb{P}^{n}$ in the Cremona groups, Manuscripta Math. 119 (2006), 25-241. 
[7] J. BLANC, Linearisation of finite abelian subgroups of the Cremona group of the plane, Groups Geom. Dyn. 3 (2009), 215-266.

[8] J. BLANC, Elements and cyclic subgroups of finite order of the Cremona group, Comment. Math. Helv. 86 (2011), 469-497.

[9] S. CAnTAT, Sur les groupes de transformations birationnelles des surfaces, Ann. of Math. (2) 174 (2011), 299-340.

[10] S. CANTAT and S. LAMY, Groupes d'automorphismes polynomiaux du plan, Geom. Dedicata 123 (2006), 201-221.

[11] J. DÉSERTI, Expériences sur certaines transformations birationnelles quadratiques, Nonlinearity 21 (2008), 1367-1383.

[12] J. DÉSERTI and J. GRIVAUX, Special automorphisms of rational surfaces with positive topological entropy, Indiana Univ. Math. J. 60 (2011), 1589-1622.

[13] J. Diller and C. FAVRe, Dynamics of bimeromorphic maps of surfaces, Amer. J. Math. 123 (2001), 1135-1169.

[14] M. H. Gizatullin, Rational G-surfaces, Izv. Akad. Nauk SSSR Ser. Mat. 44 (1980), 110-144, 239.

[15] L. Godeaux, "Les transformations birationnelles du plan”, Mémor. Sci. Math., no. 122, 2nd ed., Gauthier-Villars, Paris, 1953.

[16] G. HalphÉn, Sur les courbes planes du sixième degré à neuf points doubles, Bull. Soc. Math. France 10 (1882), 162-172.

[17] V. A. Iskovskin, Minimal models of rational surfaces over arbitrary fields, Izv. Akad. Nauk SSSR Ser. Mat. 43 (1979), 19-43.

[18] JU. I. MANIN, Rational surfaces over perfect fields. II, Mat. Sb. (N.S.) 72 (114) (1967), 161-192.

[19] S. Meskin, Nonresidually finite one-relator groups, Trans. Amer. Math. Soc. 164 (1972), $105-114$.

[20] E. Souche, "Quasi-isométrie et quasi-plans dans l'étude des groupes discrets”, $\mathrm{PhD}$ thesis, Université de Provence, 2001.

Universität Basel

Mathematisches Institut

Rheinsprung 21

CH-4051 Basel, Switzerland

jeremy.blanc@unibas.ch

Université Paris Diderot

Institut de Mathématiques

de Jussieu- Paris Rive Gauche

UP7D

58-56, avenue de France

Boite Courrier 7012

75205 Paris Cadex 13, France

deserti@math.univ-paris-diderot.fr 